This is a pre-print version of the paper. Please cite the final version of the paper:

G. Di Martino, D. Riccio, and I. Zinno, "SAR Imaging of Fractal Surfaces," IEEE Trans. Geosci. Remote Sens., vol. 50, no. 2, pp. 630-644, Feb. 2012. DOI: 10.1109/TGRS.2011.2161997.

IEEE Copyright notice. (C) 2011 IEEE. Personal use of this material is permitted. Permission from IEEE must be obtained for all other uses, in any current or future media, including reprinting/republishing this material for advertising or promotional purposes, creating new collective works, for resale or redistribution to servers or lists, or reuse of any copyrighted component of this work in other works. 


\title{
SAR Imaging of Fractal Surfaces
}

Gerardo Di Martino, Member, IEEE, Daniele Riccio, Senior Member, IEEE, and Ivana Zinno

Dipartimento di Ingegneria Biomedica, Elettronica e delle Telecomunicazioni

Università di Napoli Federico II

Via Claudio 21, 80125 Napoli, Italy

Tel. (+39)-0817683114, fax (+39)-0815934448

E-mail: \{gerardo.dimartino, daniele.riccio, ivana.zinno\}@unina.it

\begin{abstract}
A complete theoretical model for SAR imaging of natural surfaces is introduced in this paper.

The topography of the natural scenes is described via models derived from fractal geometry; scattering evaluations are performed via fractal scattering models appropriate to the employed fractal scene description. Scattering contributions are combined according to the SAR image impulse response function. The power spectral density of appropriate cuts of the SAR image are evaluated in closed form in terms of the surface fractal parameters.

Our theoretical model is here conceptually assessed, analytically derived, graphically validated, numerically verified and also tested on simulated SAR images.

The introduced model allows defining innovative post-processing inverse techniques to retrieve fractal parameters directly from SAR images.
\end{abstract}

\section{INTRODUCTION}

Analysis of microwave images of natural surfaces is a topic of increasing interest as a consequence of recent developments in remote sensing systems. Modern radars continuously supply us with high-resolution images of the Earth; moreover, they are also unveiling details of other planets and moons in the solar systems that were never monitored before by any other remote sensing tool. This scenario suggests developing new techniques to analyze radar images of natural areas. As a matter of fact, by means of low-resolution images it was only possible to identify large scale features of an observed scene (e.g., presence of mountains and shape of reliefs); conversely, by means of high-resolution images it is not only possible to improve the resolution at which we monitor the environment, but, at least in principle, we are now able to extract value added information of natural areas, presenting a much more precise physical meaning, thus providing physical-based information that cannot be trivially deduced from the input data. This activity can be very useful for a wide range of applications, e.g. prevention and monitoring of environmental 
disasters [1], [2], land classification (extraction of morphological features, land use etc.) [3], rural planning, and so on.

In this paper we deal with the fundamental issue of recovering value added information from the analysis of the behavior of single amplitude-only SAR images of natural scenes; thus, our method is conceived for supporting almost real-time applications in Earth monitoring and analysis from any interplanetary mission. We propose an analytical method for estimation of surface roughness fractal parameters based on the power spectral density behavior of SAR images along the range direction. Such a method uses both reliable electromagnetic-based scattering models and radar models to get the value added information from the SAR images.

In the existing literature there is a general lack of algorithms allowing the estimation of meaningful topographical parameters of natural surfaces from their radar image. This is due to the absence of a reliable direct model for microwave imaging of natural surfaces. A candidate direct model should originate automatic inverse procedures, that should not require supervision of a SAR expert. In addition the inverse procedure should be general-purpose, i.e. applicable to any type of SAR images, thus coping with the new generation of SAR sensors (e.g. Cosmo-SkyMed, TerraSAR-X) that exhibits extremely varied characteristics in terms of resolutions, configurations and operational modes (strip-map, spot-light, scansar); therefore, the available images can be each very different, making the information extraction procedure not immediate nor trivial. Consequently, none of the existing approaches to the problem can be assumed to be reliable for a general-purpose application [4]. More specifically, most of the already published works proposing a theoretical approach to this argument suffer from an inadequate choice of the scattering functions used to describe the electromagnetic phenomenon as unveiled by the irradiation diagrams of heuristic type that are usually considered [5]-[8]. Alternatively, some works adopt empirical approaches to retrieve significant parameters of the observed surface starting from the texture of the relevant radar image; but the lack of a physical analytical model of SAR images to be used for inversion purposes leads these works to lose in generality and applicability, requiring supervision on behalf of an expert [9], [10]. Other works model the image formation mechanism via a non minimal number of multiple-scale parameters and so, once more, are not definitively well suited as a basis for the development of inversion techniques [11], [12].

The main objective of this paper is to provide, for natural surfaces, a radar imaging model, which is stochastic and analytical. As a matter of fact, the SAR image of any natural area can be seen as the image of an element of the ensemble (a particular realization) of the stochastic process describing the observed surface: this viewpoint is convenient because we are mainly interested in the knowledge of compact statistical parameters of a natural surface, i.e. the parameters of the 
stochastic process to which the surface belongs as an element of the ensemble, rather than in its complete deterministic behavior, which is specific of the particular realization of the stochastic process of interest. In other words, for many applications involving spatial scales not very large with respect to the image resolution, it is more interesting to know some compact parameters (dimensional numbers) describing the surface roughness (e.g., fractal dimension and topothesy, or standard deviation and correlation length) more than its deterministic point-by-point behavior (i.e., a function of two independent variables). To accomplish this task, we need to evaluate the statistical characterization of the acquired image and relate it to that of the observed surface. In this paper the second order statistics of the image are evaluated in closed form, thus providing the basis for the enforcement of inversion techniques, leading to the estimation of the surface parameters directly from the radar image.

A reliable radar image modeling requires appropriate descriptions for both the observed surface and the backscattered field. Fractal models are widely recognized as the best ones to qualitatively and quantitatively describe the geometry of natural surfaces with a minimum number of independent parameters [13]-[15]. In addition to these geometrical models, fractal scattering models have been developed in order to properly represent the interaction between the electromagnetic signal and the fractal surface [16]-[18]. Therefore, we use a completely fractal approach for both geometrical and electromagnetic issues.

In the next section we present the rationale of the imaging model, i.e., we derive the relation linking the SAR image to the radar reflectivity and to the electromagnetic backscattered field. In particular, we find that the reflectivity, in the small slope regime for the surface, is linearly dependent only on the ground range partial derivative of the surface. Therefore, in Section II, the link between the SAR image and the derivative of the imaged surface is provided. In Section III the geometrical and electromagnetic models used in the paper are introduced. We describe the model used for the observed surface, i.e. the fractional Brownian motion ( $\mathrm{fBm}$ ) fractal model, and we evaluate the analytical expression of its topographic derivative process relevant to the ground range direction. Then, we evaluate in closed form the power spectral densities of this topographic derivative process of two cuts of the surface directed respectively along azimuth and ground range directions. Finally, we deal with the electromagnetic problem, presenting the fundamentals of the adopted scattering model, the fractal Small Perturbation Method (SPM), and, exploiting the general results obtained in Section II, we evaluate the reflectivity function as a function of the partial derivatives of the considered surface in the SPM case. In Section IV, by exploiting the results obtained in the previous sections, we present the complete imaging model: we provide the complete 
statistical characterization of the SAR image in terms of the surface fractal parameters. In particular, we find that the power spectral density (PSD) of the ground range cut of the amplitude SAR image exhibits, in an appropriate range of spatial frequencies, a power law behavior, while that of the azimuth cut has a more involved expression. Key considerations about the retrieving of the fractal parameters directly from the amplitude SAR image are also presented. In order to validate the theoretical results a large numerical setup is presented in Section V. In particular, a completely fractal elaboration chain, that makes also use of tools previously developed by some of the authors of this paper [19], is worked out: a canonical fractal surface of controlled fractal parameters is generated to provide the input to a SAR simulator [20]. The simulator supplies the relevant SAR raw signal, which, after standard processing, provides the simulated SAR image. We consider both the case of absence of speckle and the case of speckled images. To all these images we apply an algorithm, based on a linear regression on the range spectrum of the image, to retrieve the topographic fractal dimension of an observed region. Experimental results, obtained under the hypotheses formulated in the theoretical sections, show a very good agreement with the analytical ones. Furthermore, several fractal maps obtained by extracting the local fractal dimension from a canonical simulated SAR image are presented in Section V. Significant conclusions are reported in Section VI.

\section{IMAGING MODEL}

The direct imaging model links the morphological features (topography at a wide range of scales) and the dielectric properties of a surface (inputs) to the relevant SAR image (output).

In this section we present the direct imaging model for a SAR sensor. We split the overall model into two major elements. The first element links the SAR image to the scene reflectivity; the second element links the reflectivity to the scene parameters via a scattering model. In this section a continuous representation for the SAR image is assumed for the first part of the following analysis: this (formal) choice is done to emphasize our model behavior and stress the meaning of the obtained results. Then, the sampled counterpart of actual (bandlimited) SAR images is discussed.

In order to attain an analytical direct model for the first element, we consider a linear relationship for the SAR image, $i$, that is a filtered version of the reflectivity function depending on the resolutions of the sensor [21]:

$$
i\left(x^{\prime}, r^{\prime}\right)=\iint \gamma(x, r) \operatorname{sinc}\left[\frac{\pi}{\Delta x}\left(x^{\prime}-x\right)\right] \operatorname{sinc}\left[\frac{\pi}{\Delta r}\left(r^{\prime}-r\right)\right] d x d r
$$


where $x$ and $r$, as well as $x^{\prime}$ and $r^{\prime}$, represent azimuth and slant-range, respectively; $\gamma(x, r)$ is the two-dimensional reflectivity pattern of the scene, and includes the phase factor $\exp \left(-j \frac{4 \pi}{\lambda} r\right) ; \lambda$ is the electromagnetic wavelength, and $\Delta x$ and $\Delta r$ are the azimuth and slant-range SAR geometric resolutions, respectively.

Equation (2.1) is computed by employing the slant-range coordinate. It is convenient to reconsider it by means of its ground-range counterpart, $y$. Let us define the reflectivity map in the cylindrical coordinate system $(x, r, \theta)$. Assuming that the local incidence angle coincides with the sensor look angle, $\theta_{0}$, the ground range coordinate and the ground range resolution can be calculated by simple trigonometric computations: $y=r * \sin \theta_{0}$ and $\Delta y=\Delta r / \sin \theta_{0}$ (see Fig. 1), [21].

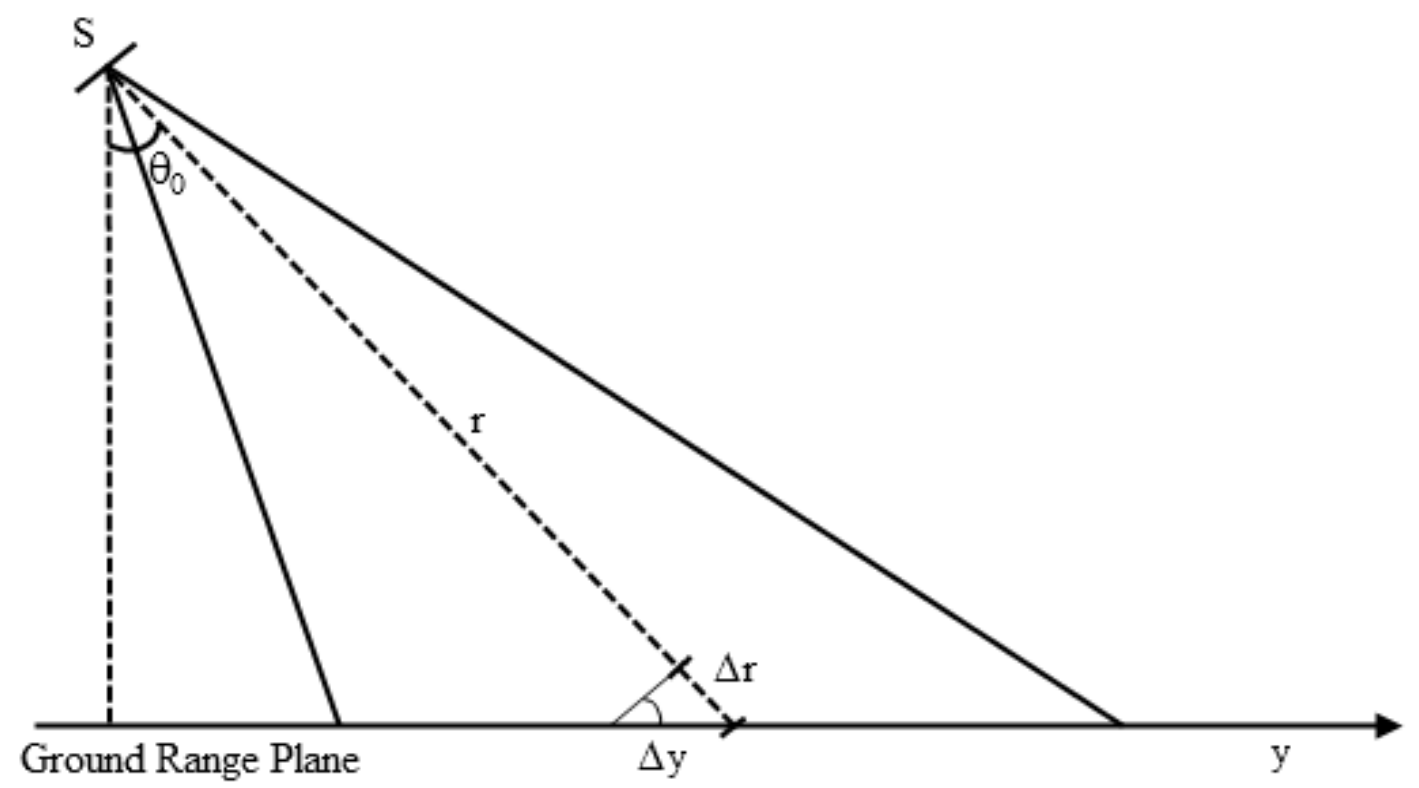

Fig. 1: Slant range vs. ground range resolutions.

Hence, in order to provide the statistical characterization of the SAR image, it is convenient to rewrite the analytical link in terms of both the image and reflectivity autocorrelation functions:

$$
\begin{aligned}
R_{i}\left(\tau_{x}{ }^{\prime}, \tau_{y}{ }^{\prime}\right)= & \left\langle i\left(x^{\prime}, y^{\prime}\right) i^{*}\left(x^{\prime}+\tau_{x}{ }^{\prime}, y^{\prime}+\tau_{y}{ }^{\prime}\right)\right\rangle= \\
= & \left\langle\frac{1}{\sin ^{2} \theta_{0}} \iint \gamma\left(x, \frac{y}{\sin \theta_{0}}\right) \operatorname{sinc}\left[\frac{\pi}{\Delta x}\left(x^{\prime}-x\right)\right] \operatorname{sinc}\left[\frac{\pi}{\Delta y \sin ^{2} \theta_{0}}\left(y^{\prime}-y\right)\right] d x d y\right. \\
& \left.\iint \gamma^{*}\left(\bar{x}, \frac{\bar{y}}{\sin \theta_{0}}\right) \operatorname{sinc}\left[\frac{\pi}{\Delta x}\left(x^{\prime}+\tau_{x}^{\prime}-\bar{x}\right)\right] \operatorname{sinc}\left[\frac{\pi}{\Delta y \sin ^{2} \theta_{0}}\left(y^{\prime}+\tau_{y}^{\prime}-\bar{y}\right)\right] d \bar{x} d \bar{y}\right\rangle=
\end{aligned}
$$




$$
\begin{aligned}
= & \frac{1}{\sin ^{2} \theta_{0}} \iint d x d \tau_{x} \iint d y d \tau_{y} R_{\gamma}\left(\tau_{x}, \frac{\tau_{y}}{\sin \theta_{0}}\right) \operatorname{sinc}\left[\frac{\pi}{\Delta x}\left(x^{\prime}-x\right)\right] \\
& \operatorname{sinc}\left[\frac{\pi}{\Delta x}\left(x^{\prime}-x+\tau_{x}^{\prime}-\tau_{x}\right)\right] \operatorname{sinc}\left[\frac{\pi\left(y^{\prime}-y\right)}{\Delta y \sin ^{2} \theta_{0}}\right] \operatorname{sinc}\left[\frac{\pi\left(y^{\prime}-y+\tau_{y}^{\prime}-\tau_{y}\right)}{\Delta y \sin ^{2} \theta_{0}}\right] .
\end{aligned}
$$

where the substitutions $\bar{x}=\tau_{x}+x$ and $\bar{y}=\tau_{y}+y$ have been considered and the stationarity of $\gamma$ (that will be proved in the next section) has been assumed.

Assuming:

$$
\int d \xi \operatorname{sinc}\left[a\left(\xi^{\prime}-\xi\right)\right] \operatorname{sinc}\left[a\left(\xi^{\prime}-\xi+\tau_{\xi}^{\prime}-\tau_{\xi}\right)\right]=R_{\operatorname{sinc}}\left[a\left(\tau_{\xi}-\tau_{\xi}^{\prime}\right)\right]
$$

where, in order to obtain the expression at the second member, a substitution of variable has been performed. Hence we get:

$$
\begin{aligned}
R_{i}\left(\tau_{x}{ }^{\prime}, \tau_{y}{ }^{\prime}\right)= & \frac{1}{\sin ^{2} \theta_{0}} \iint d \tau_{x} d \tau_{y} R_{\gamma}\left(\tau_{x}, \frac{\tau_{y}}{\sin \theta_{0}}\right) R_{\operatorname{sinc}}\left[\frac{\pi}{\Delta x}\left(\tau_{x}-\tau_{x}^{\prime}\right)\right] \\
& R_{\operatorname{sinc}}\left[\frac{\pi}{\Delta y \sin ^{2} \theta_{0}}\left(\tau_{y}-\tau_{y}^{\prime}\right)\right]
\end{aligned}
$$

Equation (2.4) provides the link, expressed in azimuth/ground-range coordinates, between the autocorrelation of the SAR image and the autocorrelation of the continuous reflectivity function. Some considerations are now in order:

- the image autocorrelation function is expressed by means of convolution integrals, so it is convenient to work in the wavenumber domain;

- in this way, since we demonstrate in the next section that the reflectivity is wide sense stationary, the Fourier transform of Eq. (2.4), which is the SAR image PSD, can be expressed as the reflectivity PSD $\left(S_{\gamma}\right)$ multiplied by the Fourier transforms of $R_{\text {sinc }}$;

- the Fourier transforms of $R_{\operatorname{sinc}}$ functions in Eq. (2.4) are rectangular functions that depend on the sensor resolutions: so we can directly consider a version of $S_{\gamma}$ filtered by means of the aforementioned rectangular functions (see Section IV).

The second element of our model, the reflectivity pattern $\gamma$, taking into account the scattering mechanism relevant to the surface, deserves a specific comment. The rationale to get Eq. (2.1) is based on a linear model postulating superposition of radar returns (reflectivity) for each point of the surface. However, the scattering process is not a point-wise one; it could be seen as a 
point-wise phenomenon only under some very specific conditions, e.g., Geometrical Optics, whose approximations do not generally hold for microwave images. Actually, in SAR images the radar returns pertaining to the same resolution cell are merged as dictated by the SAR impulse response, whereas those from different resolution cells are separated. Therefore, it is convenient to consider the contributions backscattered from different resolution cells as independent from each other, and the reflectivity pattern evaluation can be performed separately for each resolution cell.

Accordingly, we express the reflectivity pattern in terms of the Radar Cross Section (RCS) $\sigma$, as [21]:

$$
|\gamma|^{2}=\frac{\sigma}{\Delta x \Delta y}=\sigma^{0}
$$

wherein $\sigma^{0}$, the backscattering coefficient or Normalized Radar Cross Section (NRCS), represents the value of $\sigma$ normalized to the SAR resolution area.

Whatever the electromagnetic model used to evaluate the backscattering coefficient is, some general results can be obtained. The backscattering coefficient expression is linked to the surface slope via the local incidence angle $\theta$, i.e. the angle between the observation direction and the normal to the local mean plane approximating the considered surface within the resolution cell [6], [17]. Then:

$$
|\gamma|=\sqrt{\sigma^{0}}=f(\theta(p, q))
$$

where the function $f$ takes a different form depending on the selected solution to the scattering problem. Let $q$ and $p$ be the partial derivatives of the surface height $z(x, y)$ along the two directions elected by the SAR sensor, azimuth and ground-range, respectively $x$ and $y$ :

$$
\begin{aligned}
& q(x, y)=\frac{\partial z(x, y)}{\partial x} \\
& p(x, y)=\frac{\partial z(x, y)}{\partial y}
\end{aligned}
$$

The local incidence angle can be formally expressed as a function of the partial derivatives $p$ and $q$, in fact its cosine can be evaluated as the scalar product between the propagation unit vector and the surface normal unit vector, i.e.: 


$$
\theta=\cos ^{-1}\left(\frac{p \sin \theta_{0}+\cos \theta_{0}}{\sqrt{p^{2}+q^{2}+1}}\right)
$$

In the hypothesis of a small slope regime for the surface, a McLaurin series expansion of the function $f(\theta(p, q))$ in Eq. (2.6) with respect to $p$ and $q$ can be performed: to the first order, we obtain a linear function of the partial derivative $p$ only; as a matter of fact, from Eq. (2.9), it is clear that the derivative of $\theta$ with respect to $q$ is proportional to $q$ itself, implying that the linear term in $q$ of the McLaurin expansion is zero.

Therefore, the modulus of the reflectivity function $|\gamma(x, y)|$, is, in a first order approximation, linearly linked only to the partial derivative $p$ of the surface:

$$
\begin{gathered}
|\gamma(x, y)|=f(\theta(p, q))=a_{0}+a_{1} p(x, y)+o(p, q), \\
a_{0}=f(\theta(p=0, q=0)) \quad a_{1}=\left.\frac{\partial f(\theta(p, q))}{\partial p}\right|_{\substack{p=0 \\
q=0}},
\end{gathered}
$$

$a_{0}$ and $a_{1}$ being the coefficients of the McLaurin series expansion, whose expressions depend on the specific scattering model that is adopted. In particular, these coefficients are function of the look angle of the sensor, which is then an important parameter for the determination of the validity limits of the proposed linear model. Finally, we note that the obtained result highlights a key property of SAR - and, more in general, of side-looking radars - imaging behavior, showing a clear mathematical definition of a preferential imaging direction due to their particular acquisition geometry.

The result in Eq. (2.10) is valid independently of the selected scattering function $f(\theta(p, q))$, hence it holds for whatever electromagnetic model (which can be evaluated analytically in closed form) is chosen and it presents reasonably a general validity, given the small slope regime for the observed surface. In Section III.2, the coefficients of the McLaurin series expansion, $a_{0}$ and $a_{1}$ are evaluated in closed form for a specific scattering function, the SPM one.

The above reported analysis can be assumed as a clear and valid foundation to assess the statistical characterization of the image. Usually, this characterization must be derived from a single amplitude SAR image and we cannot set aside the speckle phenomenon, the multiplicative noise affecting SAR images: for the sake of a theoretical analysis, we can consider the speckle as part of the reflectivity. As a matter of fact, radar single-look images hold small scale spatial properties (corresponding to high wavenumber spectral properties) dominated by the speckle effect. A 
theoretical and quantitative analysis of the speckle is not the objective of this paper, and it will be one of the main future developments of this work. Anyway, some hints are provided in Section V.2. Now a comment about the bandwidths of the reflectivity and of the SAR acquisition system is in order.

Due to the scattering mechanism, the reflectivity function holds a finite (spatial frequency) bandwidth: the minimum wavenumber being related to the (inverse of the) size of the illuminated area, the maximum one being related to the (inverse of the) electromagnetic wavelength. Due to the role played by the SAR system, the SAR image holds a different but still finite bandwidth: the minimum wavenumber being related to the (inverse of the) size of the considered area, the maximum one being related to the (inverse of the) SAR resolution. Therefore, the image autocorrelation function depends on the SAR resolutions: so we look for the analytical relationship between the reflectivity function (sampled according to SAR resolutions) and the parameters of the observed surface. Hence, we ought to work with a two scale model for the surface description: the observed surface is locally approximated by square plane facets with dimension equal to that of the resolution cells; over these plane facets a microscopic roughness is superimposed so that the electromagnetic field backscattered from each resolution cell can be evaluated. Hence, the individual returns from each resolution cell are dictated by the microscopic scale (below resolution cell) roughness, while the overall image texture is related to the macroscopic scale (above resolution cell) roughness.

\section{FRACTAL MODELS}

In order to obtain the spectral behavior of the reflectivity pattern as acquired by a sensor of prescribed resolution, it is fundamental to describe both the geometry of the surface and the electromagnetic field backscattered from it.

\section{III.1 Geometrical model}

It is widely recognized that fractal geometry is the best candidate to describe the irregularity and the roughness of natural scenes. Among the fractal models, we make use of the regular stochastic fractional Brownian motion (fBm) process to describe natural surfaces [13]-[17]. It can be defined in terms of the corresponding increment process: the two-dimensional stochastic process $z(x, y)$ describes an isotropic (mathematical) fBm surface if, for every $x, y, x^{\prime}, y^{\prime}$, all belonging to $R$, the increment process $z(x, y)-z\left(x^{\prime}, y^{\prime}\right)$ satisfies the following relation: 


$$
\begin{gathered}
\operatorname{Pr}\left\{z(x, y)-z\left(x^{\prime}, y^{\prime}\right)<\bar{\zeta}\right\}=\frac{1}{\sqrt{2 \pi} s \tau^{H}} \int_{-\infty}^{\bar{\zeta}} \exp \left(-\frac{\zeta^{2}}{2 s^{2} \tau^{2 H}}\right) d \zeta, \\
\tau=\sqrt{\left(x-x^{\prime}\right)^{2}+\left(y-y^{\prime}\right)^{2}}
\end{gathered}
$$

wherein $H$ is the Hurst coefficient $(0<H<1)$ and $s$ is the incremental standard deviation of surface, measured in $\left[\mathrm{m}^{(1-H)}\right]$, i.e. the standard deviation evaluated for increments at unitary distance. The parameter $s$ is related to a characteristic length of the fBm surface, called topothesy $T$ [m] by the relation $s=T^{(1-H)}$. Topothesy is the distance over which chords joining points on the surface have a root mean square (rms) slope equal to unity.

The Hurst coefficient, $H$, is related to the fractal dimension through the expression:

$$
D=3-H
$$

Note that, while the $\mathrm{fBm}$ process is non-stationary, the increment process is wide sense stationary [17].

The PSD of the topographic two-dimensional isotropic $\mathrm{fBm}$ process - in spite of the complexity of the derivation of its expression, involving the evaluation of the spectrum of a nonstationary process, for instance obtained through Wigner-Ville or wavelet analysis [17], [22], [23] exhibits an appropriate power-law behavior:

$$
S(\boldsymbol{k})=S_{0} \boldsymbol{k}^{-\alpha}
$$

wherein $\boldsymbol{k}=\sqrt{k_{x}^{2}+k_{y}^{2}}$ is the wavenumber and $k_{x}$ and $k_{y}$ are its components along the azimuth and ground range directions respectively; $S_{0}$ and $\alpha$ are the spectral parameters, related to the spatial ones by the following relationships [17]:

$$
\begin{gathered}
S_{0}=2^{H+1} \Gamma^{2}(1+H) \sin (\pi H) \mathrm{s}^{2} \\
\alpha=2+2 H=8-2 D
\end{gathered}
$$

$\Gamma(\cdot)$ being the Gamma function.

The PSD of a topographic one-dimensional fBm profile (that coincides with a onedimensional cut of an $\mathrm{fBm}$ surface) is also introduced: 


$$
S(k)=S_{0}^{\prime} k^{-\alpha^{\prime}},
$$

wherein $k$ is the wavenumber and $S_{0}^{\prime}$ and $\alpha^{\prime}$ are the spectral parameters in the one-dimensional case:

$$
\begin{gathered}
S_{0}^{\prime}=\frac{\pi H}{\cos (\pi H)} \frac{1}{\Gamma(1-2 H)} s^{2}, \\
\alpha^{\prime}=1+2 H=5-2 D .
\end{gathered}
$$

Note that the spectra of natural surfaces present a power-law behavior over a wide range of spatial scales [24]-[26].

Therefore, the application of the model to observable quantities (surfaces), leads us to use physical (bandlimited) fBms. By definition for physical (bandlimited) fBms the above relationships are still valid in the corresponding spatial scales or spectral bandwidths.

In Section II we have shown that the stochastic characterization of the SAR image involves use of the partial derivatives of the sensed surface. The formal derivative of an $\mathrm{fBm}$ profile is defined as fractional Gaussian noise (fGn) [23], [27], and its power spectral density is proportional to that of the fBm profile multiplied by $k^{2}$ [23], [27]:

$$
S_{f G n}(k) \propto \frac{1}{|k|^{2 H-1}}
$$

However, SAR images present a finite spatial extent and are discretized according to a nonzero lag sampling. Hence, application to SAR images requires the definition of bandlimited stochastic processes, whose analytical form depends on the specific bandlimiting procedure applied. In order to get a closed form expression for the SAR image power spectrum, it is mandatory to consider the role of the resolution cell; this is convenient also because it allows working with a twoscale model for the surface. In this context, we want to study the canonical case of a fractal surface, with the same fractal parameters, at all the scales of interest for the sensor. In fact, a SAR sensor discriminates between scales lower and greater than the resolution cell size. Therefore, in our case the surface description within the resolution cell is introduced as a microscopic fractal roughness superimposed to a plane facet (having the dimension of the resolution cell) approximating the scene of interest; the macroscopic surface description at the resolution cell scale, which is related to the applied bandlimiting procedure, is then required in order to evaluate the PSD of interest.

Actually, to cope with the non-differentiability of the fBm process, a smoothed version of the original $\mathrm{fBm}$ process can be introduced [27]; this is a filtered version of the original surface, 
obtained by multiplying it by a differentiable test function, $\varphi$ : the test function support is, for the time being, set equal to $\left[0, \varepsilon_{x}\right] \times\left[0, \varepsilon_{y}\right], \varepsilon_{x}$ and $\varepsilon_{y}$ being related to the SAR resolutions in azimuth and ground range, respectively. Thus, we set:

$$
\begin{gathered}
\varphi(x, y)=\left\{\begin{array}{cc}
\frac{1}{\varepsilon_{x} \varepsilon_{y}} & \text { if }(x, y) \in\left[0, \varepsilon_{x}\right] \times\left[0, \varepsilon_{y}\right] \\
0 & \text { otherwise }
\end{array}\right. \\
z_{\varphi}(x, y)=\iint_{-\infty}^{\infty} z\left(x^{\prime}, y^{\prime}\right) \varphi\left(x-x^{\prime}, y-y^{\prime}\right) d x^{\prime} d y^{\prime}=\frac{1}{\varepsilon_{x} \varepsilon_{y}} \int_{x-\varepsilon_{x}}^{x} \int_{y-\varepsilon_{y}}^{y} z\left(x^{\prime}, y^{\prime}\right) d x^{\prime} d y^{\prime} .
\end{gathered}
$$

A comment on the relevance of the partial derivatives of the observed surface in imaging theory is in order. These are of clear physical meaning, providing information on the asymmetry in the SAR data structure with respect to the $x$ and $y$ directions, intuitively consistent with the existence of a preferential direction of sight of SAR sensors.

In fact, as we have seen in the previous section, the reflectivity function, in a first order approximation, depends only on the partial derivative of the surface with respect to the ground range coordinate, as can be seen from Eq. (2.10). Moreover, we note that the functional $z_{\varphi}(x, y)$ presented in Eq. (3.10) can be seen as a distribution [27]. Hence, for our surface the partial derivative with respect to the ground range direction, $z_{p}\left(x, y ; \varepsilon_{y}\right)$, can be defined using the theory of distributions, i.e. moving the derivation from the process $z(x, y)$ to the test function $\varphi(x, y)$ [28], thus obtaining:

$$
\begin{aligned}
z_{p}\left(x, y ; \varepsilon_{y}, \varepsilon_{x}\right) \triangleq \frac{\partial z(x, y)}{\partial y} & =\iint_{-\infty}^{\infty} z\left(x^{\prime}, y^{\prime}\right) \frac{\partial \varphi}{\partial y}\left(x-x^{\prime}, y-y^{\prime}\right) d x^{\prime} d y^{\prime}= \\
& =\frac{1}{\varepsilon_{x} \varepsilon_{y}} \int_{x-\varepsilon_{x}-\infty}^{x} \int^{\infty} z\left(x^{\prime}, y^{\prime}\right)\left[\delta\left(y-y^{\prime}\right)-\delta\left(y-\varepsilon_{y}-y^{\prime}\right)\right] d y^{\prime} d x^{\prime}= \\
& =\frac{1}{\varepsilon_{x} \varepsilon_{y}} \int_{x-\varepsilon_{x}}^{x}\left[z\left(x^{\prime}, y\right)-z\left(x^{\prime}, y-\varepsilon_{y}\right)\right] d x^{\prime}
\end{aligned}
$$

Hence, $z_{p}\left(x, y ; \varepsilon_{y}\right)$ is linearly related to the $\mathrm{fBm}$ increment process and it is, for this reason, wide sense stationary. Therefore, the autocorrelation function of the partial derivative process $z_{p}(x, y)$ can be evaluated starting from the correlation between two increments of the fBm original 
process:

$$
\begin{aligned}
& R_{z_{p}}\left(\tau_{x}, \tau_{y} ; \varepsilon_{y}\right)=\left\langle z_{p}\left(x, y ; \varepsilon_{y}\right) z_{p}\left(x+\tau_{x}, y+\tau_{y} ; \varepsilon_{y}\right)\right\rangle= \\
& =\left\langle\frac{1}{\left(\varepsilon_{x} \varepsilon_{y}\right)^{2}} \int_{x-\varepsilon_{x}}^{x}\left[z\left(x^{\prime}, y\right)-z\left(x^{\prime}, y-\varepsilon_{y}\right)\right] d x^{\prime} \int_{x-\varepsilon_{x}}^{x}\left[z\left(x^{\prime \prime}+\tau_{x}, y+\tau_{y}\right)-z\left(x^{\prime \prime}+\tau_{x}, y+\tau_{y}-\varepsilon_{y}\right)\right]\right. \\
& =\frac{1}{\left(\varepsilon_{x} \varepsilon_{y}\right)^{2}} \int_{x-\varepsilon_{x}}^{x} \int_{x-\varepsilon_{x}}^{x}\left\langle\left[ z\left(x^{\prime}, y\right) z\left(x^{\prime \prime}+\tau_{x}, y+\tau_{y}\right)-z\left(x^{\prime}, y\right) z\left(x^{\prime \prime}+\tau_{x}, y+\tau_{y}-\varepsilon_{y}\right)+\right.\right. \\
& \left.\left.-z\left(x^{\prime}, y-\varepsilon_{y}\right) z\left(x^{\prime \prime}+\tau_{x}, y+\tau_{y}\right)+z\left(x^{\prime}, y-\varepsilon_{y}\right) z\left(x^{\prime \prime}+\tau_{x}, y+\tau_{y}-\varepsilon_{y}\right)\right]\right\rangle d x^{\prime} d x^{\prime \prime}=
\end{aligned}
$$

wherein $\tau_{x}$ and $\tau_{y}$ are space lags in the azimuth and ground-range direction, respectively:

$$
\tau_{x}=\sqrt{\left(x-x^{\prime}\right)^{2}} ; \quad \tau_{y}=\sqrt{\left(y-y^{\prime}\right)^{2}}
$$

Considering that the autocorrelation of an $\mathrm{fBm}$ is given by [17]:

$$
\left\langle z(\boldsymbol{r}) z\left(\boldsymbol{r}^{\prime}\right)\right\rangle=\frac{T^{2(1-H)}}{2}\left(|\boldsymbol{r}|^{2 H}+\left|\boldsymbol{r}^{\prime}\right|^{2 H}-\left|\boldsymbol{r}^{\prime}-\boldsymbol{r}\right|^{2 H}\right)
$$

substituting Eq. (3.14) in Eq. (3.12) we get:

$$
\begin{gathered}
R_{z_{p}}\left(\tau_{x}, \tau_{y} ; \varepsilon_{y}\right)= \\
\begin{aligned}
=s^{2} \frac{1}{\left(\varepsilon_{x} \varepsilon_{y}\right)^{2}} \int_{x-\varepsilon_{x}}^{x} \int_{x-\varepsilon_{x}}^{x}\left[\left|\tau_{x}^{2}+\left(\tau_{y}+\varepsilon_{y}\right)^{2}\right|^{H}+\left|\tau_{x}^{2}+\left(\tau_{y}-\varepsilon_{y}\right)^{2}\right|^{H}+-2\left|\tau_{x}^{2}+\tau_{y}^{2}\right|^{H}\right] d x^{\prime} d x^{\prime \prime}= \\
=s^{2} \varepsilon_{y}^{-2}\left[\left|\tau_{x}^{2}+\left(\tau_{y}+\varepsilon_{y}\right)^{2}\right|^{H}+\left|\tau_{x}^{2}+\left(\tau_{y}-\varepsilon_{y}\right)^{2}\right|^{H}-2\left|\tau_{x}^{2}+\tau_{y}^{2}\right|^{H}\right]
\end{aligned}
\end{gathered}
$$

The autocorrelation function in Eq. (3.15) allows the evaluation of the two-dimensional power spectrum. However, in imaging theory (and in particular for a SAR sensor which is characterized by different spatial resolutions along azimuth and range), a more meaningful role is played by the power density spectra of cuts (along azimuth and ground range) of the image. 
Analytical expressions for these spectra are here analytically evaluated via a Fourier Transform of the azimuth and ground range cuts of the two-dimensional autocorrelation function reported in Eq. (3.15): as a matter of fact Eq. (3.15) shows that $z_{p}$ is wide sense stationary and the WienerKintchine theorem can be applied.

- For a ground-range cut, from Eq. (3.13) we get:

$$
R_{p}\left(\tau_{y} ; \varepsilon_{y}\right)=R_{z_{p}}\left(\tau_{x}=0, \tau_{y} ; \varepsilon_{y}\right)=\frac{1}{2} s^{2} \varepsilon_{y}{ }^{2 H-2}\left[\left(\frac{\left|\tau_{y}\right|}{\varepsilon_{y}}+1\right)^{2 H}-2\left|\frac{\tau_{y}}{\varepsilon_{y}}\right|^{2 H}+\left(\frac{\left|\tau_{y}\right|}{\varepsilon_{y}}-1\right)^{2 H}\right]^{2}
$$

leading to [29], [19]:

$$
S_{p}\left(k_{y} ; \varepsilon_{y}\right)=2 s^{2} \varepsilon_{y}^{-1+2 H} \Gamma(1+2 H) \sin (\pi H)\left[1-\cos \left(\left|k_{y}\right| \varepsilon_{y}\right)\right] \frac{1}{\left(\left|k_{y}\right| \varepsilon_{y}\right)^{1+2 H}} .
$$

In this case, the autocorrelation function, $R_{p}$, and the PSD, $S_{p}$, of the derivative process match exactly with those introduced for a one-dimensional profile [19].

Moreover, it is interesting and useful, to evaluate $\tilde{S}_{p}$ defined as the limit of $S_{p}$ for $k_{y} \varepsilon_{y} \rightarrow 0$ :

$$
\tilde{S}_{p}\left(k_{y}\right)=s^{2} \Gamma(1+2 H) \sin (\pi H) \frac{1}{\left|k_{y}\right|^{2 H-1}}
$$

In Eq. (3.18) $\tilde{S}_{p}\left(k_{y}\right)$ provides an asymptotic evaluation and is amenable to meaningful interpretation and application: for every $k_{y}$ it is analytically obtained by reducing the support of the test function; alternatively, for every $\varepsilon_{y}$, i.e., for actual radar resolutions, it approximates the low spatial wavenumbers regime of the estimated PSD.

- For the azimuth cut, from Eq. (3.15) we get:

$$
R_{p}\left(\tau_{x} ; \varepsilon_{y}\right)=R_{z_{p}}\left(\tau_{x}, \tau_{y}=0 ; \varepsilon_{y}\right)=s^{2} \varepsilon_{y}{ }^{-2}\left[\left|\tau_{x}^{2}+\varepsilon_{y}^{2}\right|^{H}-\left|\tau_{x}\right|^{2 H}\right]
$$

Evaluation of the corresponding PSD was never done before and is introduced hereafter in this paper. Also in this case, it requires resorting to generalized Fourier Transforms; for the first term of Eq. (3.19) we get [29]: 


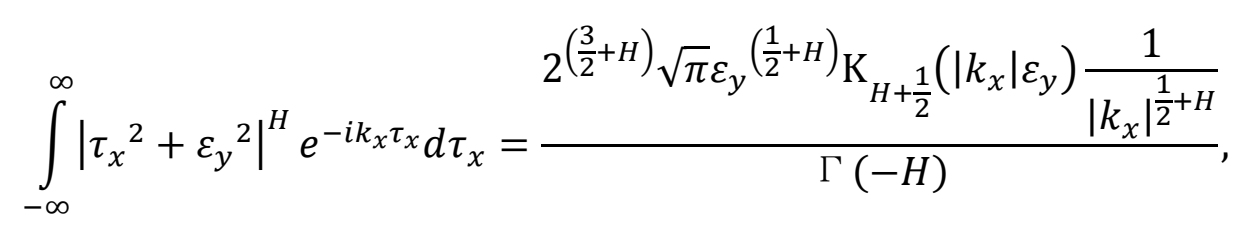

and for the second term we obtain [29], [19]:

$$
\int_{-\infty}^{\infty}\left|\tau_{x}\right|^{2 H} e^{-i k_{x} \tau_{x}}=2 \Gamma(1+2 H) \sin (\pi H) \frac{1}{\left|k_{x}\right|^{1+2 H}}
$$

Thus, we can evaluate in closed form the PSD of $z_{p}(x, y)$ for an azimuth cut of the surface:

$S_{p}\left(k_{x} ; \varepsilon_{y}\right)=s^{2} \varepsilon_{y}{ }^{-1+2 H}\left[\frac{2^{\left(\frac{3}{2}+H\right)} \sqrt{\pi} \mathrm{K}_{H+\frac{1}{2}}\left(\left|k_{x}\right| \varepsilon_{y}\right) \frac{1}{\left(\left|k_{x}\right| \varepsilon_{y}\right)^{\frac{1}{2}+H}}}{\Gamma(-H)}+2 \Gamma(1+2 H) \sin (\pi H) \frac{1}{\left(\left|k_{x}\right| \varepsilon_{y}\right)^{1+2 H}}\right]$

where $\mathrm{K}_{v}(\cdot)$ is the modified Bessel function of second type of fractional order $v$.

In order to point out the asymptotical spectral behavior of the aforementioned spectrum, we can express the function $\mathrm{K}_{H+\frac{1}{2}}\left(\left|k_{x}\right| \varepsilon_{y}\right)$ through a power series expansion around the value $k_{x}=0$ stopped to the first order [29]:

$$
\begin{aligned}
K_{H+\frac{1}{2}}\left(\left|k_{x}\right| \varepsilon_{y}\right) & =2^{-\frac{3}{2}-H}\left(\left|k_{x}\right| \varepsilon_{y}\right)^{\frac{1}{2}+H}\left[1+\frac{\left(\left|k_{x}\right| \varepsilon_{y}\right)^{2}}{(6+4 H)}\right] \Gamma\left(-\frac{1}{2}-H\right) \\
& +2^{-\frac{1}{2}+H}\left(\left|k_{x}\right| \varepsilon_{y}\right)^{-\frac{1}{2}-H}\left[1+\frac{\left(\left|k_{x}\right| \varepsilon_{y}\right)^{2}}{(2-4 H)}\right] \Gamma\left(\frac{1}{2}+H\right) .
\end{aligned}
$$

Therefore, substituting Eq. (3.23) in Eq. (3.22) we obtain the following expression of the spectrum: 


$$
\begin{aligned}
\tilde{S}_{p}\left(k_{x} ; \varepsilon_{y}\right)= & s^{2} \varepsilon_{y}{ }^{2 H-1}\left\{\frac{\sqrt{\pi} \Gamma\left(-\frac{1}{2}-H\right)}{\Gamma(-H)}+\frac{\sqrt{\pi} \Gamma\left(-\frac{1}{2}-H\right)}{2(2 H) \Gamma(-H)}\left(\left|k_{x}\right| \varepsilon_{y}\right)^{2}\right. \\
& +\frac{\sqrt{\pi} 2^{1+2 H} \Gamma\left(\frac{1}{2}+H\right)}{(1-2 H) \Gamma(-H)} \frac{1}{\left(\left|k_{x}\right| \varepsilon_{y}\right)^{2 H-1}} \\
& \left.+\left[\frac{\sqrt{\pi} 2^{1+2 H} \Gamma\left(\frac{1}{2}+H\right)}{\Gamma(-H)}+2 \Gamma(1+2 H) \sin (\pi H)\right] \frac{1}{\left(\left|k_{x}\right| \varepsilon_{y}\right)^{2 H+1}}\right\} .
\end{aligned}
$$

Expression (3.24) can be simplified by considering that [29]:

$$
\frac{\sqrt{\pi} \Gamma\left(\frac{1}{2}+H\right)}{\Gamma(-H)}=-2^{-2 H} \Gamma(1+2 H) \sin (\pi H)
$$

so Eq. (3.24) can be written as:

$$
\begin{aligned}
\tilde{S}_{p}\left(k_{x} ; \varepsilon_{y}\right)= & s^{2} \varepsilon_{y}{ }^{2 H-1}\left\{\frac{\sqrt{\pi} \Gamma\left(-\frac{1}{2}-H\right)}{\Gamma(-H)}+\frac{\sqrt{\pi} \Gamma\left(-\frac{1}{2}-H\right)}{4 H \Gamma(-H)}\left(\left|k_{x}\right| \varepsilon_{y}\right)^{2}\right. \\
& \left.-\frac{2 \Gamma(1+2 H) \sin (\pi H)}{2-4 H} \frac{1}{\left(\left|k_{x}\right| \varepsilon_{y}\right)^{2 H-1}}\right\} .
\end{aligned}
$$

The introduced formulas deserve some significant considerations. First of all, differently from the case of the ground-range cut, the spectrum of the partial derivative process for the azimuth cut does not show a power law behavior, not even asymptotically.

Owing to the radar preferential direction of sight, in the case of a range profile we are considering the derivative along the same direction of the performed cut; this implies that the spectrum of the derivative process inherits the correlation properties of successive increments of the $\mathrm{fBm}$ profile. Conversely, for an azimuth profile such considerations are not valid anymore: in this case we are considering the derivative in the ground range direction whereas the profile originates from an azimuth cut of the surface, so the properties of the derivative process is not directly linked to the profile behavior. 
In order to evaluate the reflectivity pattern $\gamma$, we need an appropriate scattering model taking into account the specific geometrical characterization used for the observed scene. Hence, we must consider the interaction between the electromagnetic field and the fractal surface by means of an appropriate fractal scattering model tailored to the case at hand. The candidate scattering model should lead to a closed form solution for the reflectivity function (and for the backscattering coefficient). For rough surfaces only approximate solutions are available, each solution being valid under appropriate roughness and illumination conditions [16]-[18]. In this paper we use the SPM which provides the simplest expression for the NRCS and shows a range of validity adequate to SAR applications.

The NRCS for the SPM model in the fractal case is [16], [17]:

$$
\sigma_{m n}^{0}=4 \kappa^{3} \cos ^{4} \theta\left|\beta_{m n}\right|^{2} \frac{S_{0}}{(2 \kappa \sin \theta)^{2+2 H}}
$$

wherein $\kappa$ is the electromagnetic wavenumber; $\beta_{m n}$, accounting for the incident and reflected fields polarization, is a function of both the dielectric constant of the surface and the local incidence angle $\theta$ [17]; $S_{0}$ and $H$ are the surface fractal parameters introduced in the first part of this section. Note that with the considered model we are able to deal only with the co-polarized case.

Now, it is possible to use the results presented in Section II in order to obtain an expression of the reflectivity function as a function of the partial derivatives of the surface. In particular, substituting the expression of $\cos \theta$ provided in Eq. (2.9) and the corresponding expression of $\sin \theta$, into Eq. (3.27) and, taking into account that the term $\left|\beta_{m n}\right|^{2}$ can be considered constant with $\theta$ in the angular interval of interest in the co-polarized case, the NRCS can be then expressed as:

$$
\sigma^{0}=A_{0}\left(\frac{\left(\cos \theta_{0}+p \sin \theta_{0}\right)^{2}}{p^{2}+q^{2}+1}\right)^{2}\left(\frac{\left(\sin \theta_{0}-p \cos \theta_{0}\right)^{2}+q^{2}}{p^{2}+q^{2}+1}\right)^{-(1+H)}
$$

wherein

$$
A_{0}=\frac{S_{0} \kappa^{1-2 H}\left|\beta_{m n}\right|^{2}}{2^{2 H}}
$$

Therefore $|\gamma(x, y)|$, which is related to $\sigma^{0}$ by Eq. (2.5), can be evaluated as: 


$$
\begin{aligned}
|\gamma(x, y)| & =f(\theta(p, q))= \\
& =\sqrt{A_{0}}\left(\frac{\left(\cos \theta_{0}+p \sin \theta_{0}\right)^{2}}{p^{2}+q^{2}+1}\right)\left(\frac{\left(\sin \theta_{0}-p \cos \theta_{0}\right)^{2}+q^{2}}{p^{2}+q^{2}+1}\right)^{\frac{-(1+H)}{2}}
\end{aligned}
$$

Performing the McLaurin series expansion of the expression in Eq. (3.30) we obtain the coefficients $a_{0}$ and $a_{1}$ (see Eq. 2.10) relevant to the SPM scattering function:

$$
\begin{aligned}
|\gamma(x, y)| & \cong a_{0}+a_{1} p= \\
& =\sqrt{A_{0}}\left\{\cos ^{2} \theta_{0} \sin ^{-(1+H)} \theta_{0}+\cos \theta_{0} \sin ^{-H} \theta_{0}\left[2+(1+H) \cos ^{2} \theta_{0} \sin ^{-2} \theta_{0}\right] p\right\}
\end{aligned}
$$

wherein $p$ is characterized in the first part of this section. Therefore, in the case of interest, the coefficients $a_{0}$ and $a_{1}$, and in turn the validity limits of the proposed model, depend on the considered look angle and on the fractal parameters of the observed surface.

\section{STOCHASTIC CHARACTERIZATION OF THE SAR IMAGE}

Exploiting the results obtained in the previous sections, the complete statistical characterization of a SAR image is presented in this section.

According to the theoretical results presented in the previous sections, provided that the slopes of the surface are sufficiently low, the image is linearly dependent on the partial derivative process $z_{p}$, whose expression is given in Eq. (3.11). Hence the image inherits the same statistical characterization of the process $z_{p}(x, y)$, i.e. it is Gaussian distributed with $\mu=a_{0}$ and $\sigma=a_{1} s \Delta y$, as we can deduce combining Eq. (2.10) and (3.11).

A discussion is now in order on the role of $\varepsilon_{x}$ and $\varepsilon_{y}$, defining the support of the kernel $\varphi$ mentioned in the previous section, which formally determines the effective bandwidth of the imaging system whenever applied to the fractal surfaces. As far as the bandwidth is concerned, our model implies dealing with two, somehow implicit, band-limiting procedures that can be conveniently formalized as two filtering steps that we now explicitly discuss. First of all, the electromagnetic field impinging on the rough surface performs a low-pass filtering on the surface according to the electromagnetic wavelength, $\lambda$. Then, the obtained smoothed process is filtered according to the sensor impulse response Eq. (2.1), and spatial scales lower than the resolution one are discarded. In our case, assuming $x$ and $y$ as coordinates, in azimuth and ground-range directions, respectively, and $\Delta x$ and $\Delta y$ as the corresponding sensor resolutions, we can consider, being $\Delta x, \Delta y$ 
$>>$, directly the second filtering step and we can take $\varepsilon_{x}$ and $\varepsilon_{y}$ coincident with the azimuth and ground-range resolutions.

Considering the expression of the SAR image autocorrelation function (see Eq. (2.2)), and applying the Wiener-Kintchine theorem, we can now provide the power density spectra for a range, $S_{i}\left(k_{y}\right)$, and an azimuth, $S_{i}\left(k_{x}\right)$, cut of the image in closed form:

$$
\begin{gathered}
S_{i}\left(k_{y}\right)=a_{1}^{2} S_{p}\left(k_{y} ; \Delta y\right) \operatorname{rect}\left[\frac{\Delta y \sin ^{2} \vartheta k_{y}}{\pi}\right] \\
S_{i}\left(k_{x}\right)=a_{1}^{2} S_{p}\left(k_{x} ; \Delta y\right) \operatorname{rect}\left[\frac{\Delta x k_{x}}{\pi}\right]
\end{gathered}
$$

Recalling that for the closed form expression obtained in the previous section for the power density spectra $S_{p}\left(k_{y} ; \Delta y\right)$ and $S_{p}\left(k_{x} ; \Delta y\right)$ in Eqs. (3.17), (3.22) and for their asymptotic formulations in Eqs. (3.18), (3.26) a meaningful dependence on the fractal parameters of the observed surface was found, we can now draw some significant considerations. As a matter of fact, image range cuts - in an appropriate range of frequencies, i.e. $k_{y} \Delta y \ll 2 \pi$ - exhibit spectra with a linear behavior in a log-log plane, as shown in Eq. (3.18), thus allowing implementation of linear regression techniques to retrieve the fractal parameters of the observed scene directly from the corresponding radar image. In particular, by comparing Eq. (3.18) with the expression of the PSD of a one-dimensional cut of the surface in Eq. (3.6), we infer that, in the log-log plane, the slope of the range spectrum of a SAR image is equal to that of the imaged surface whereas the surface Hurst coefficient is decreased by one.

Conversely, for azimuth cuts, as we infer from Eq. (3.26), also for very low frequencies, the above discussion does not hold any longer; the azimuth image spectrum is quite involved and the retrieving techniques should be non linear ones.

For a visual inspection of the obtained theoretical results, in Figs. 2-5 the azimuth (dashdot line) and range (continuous line) spectra of an image are shown in a $\log (k)-\log (|\mathrm{S}(k)|)$ plane, where the same values of $s=0.1 \mathrm{~m}^{1-H}$ and $a_{1}=1$ and different $H$ values (marked in the captions) are considered; for comparison purposes, also the behavior of the spectrum of a cut of the original surface relevant to Eq. (3.6) (dashed line) is reported in the same graphs. In order to compare the spectra behaviors wavenumbers are normalized to the value of the considered resolution. 


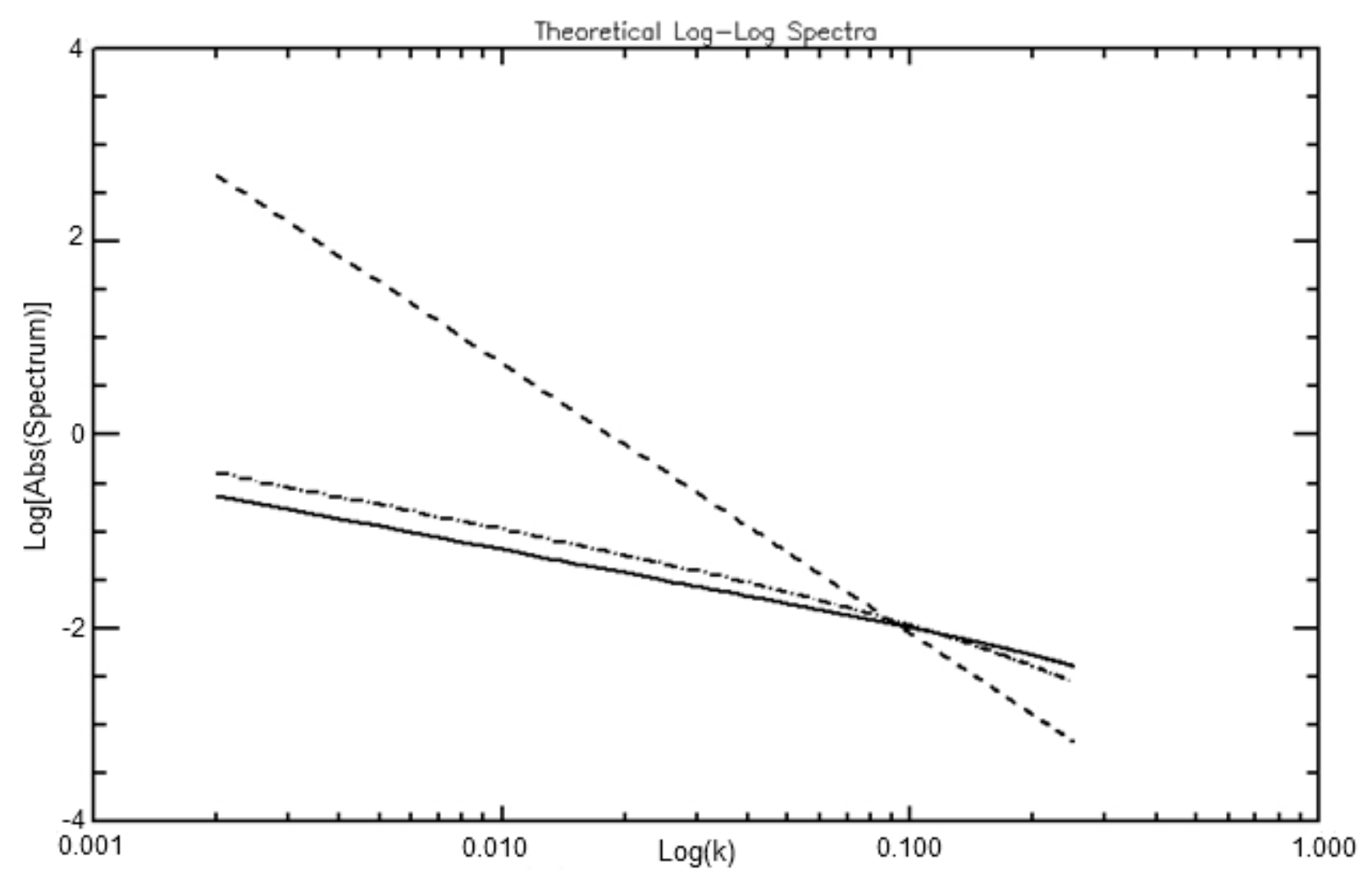

Fig. 2: Theoretical log-log plots of range (continuous line) and azimuth (dash-dot line) image cuts PSD; the dashed line represents the surface cut PSD. All the graphs are relevant to $H=0.9$.

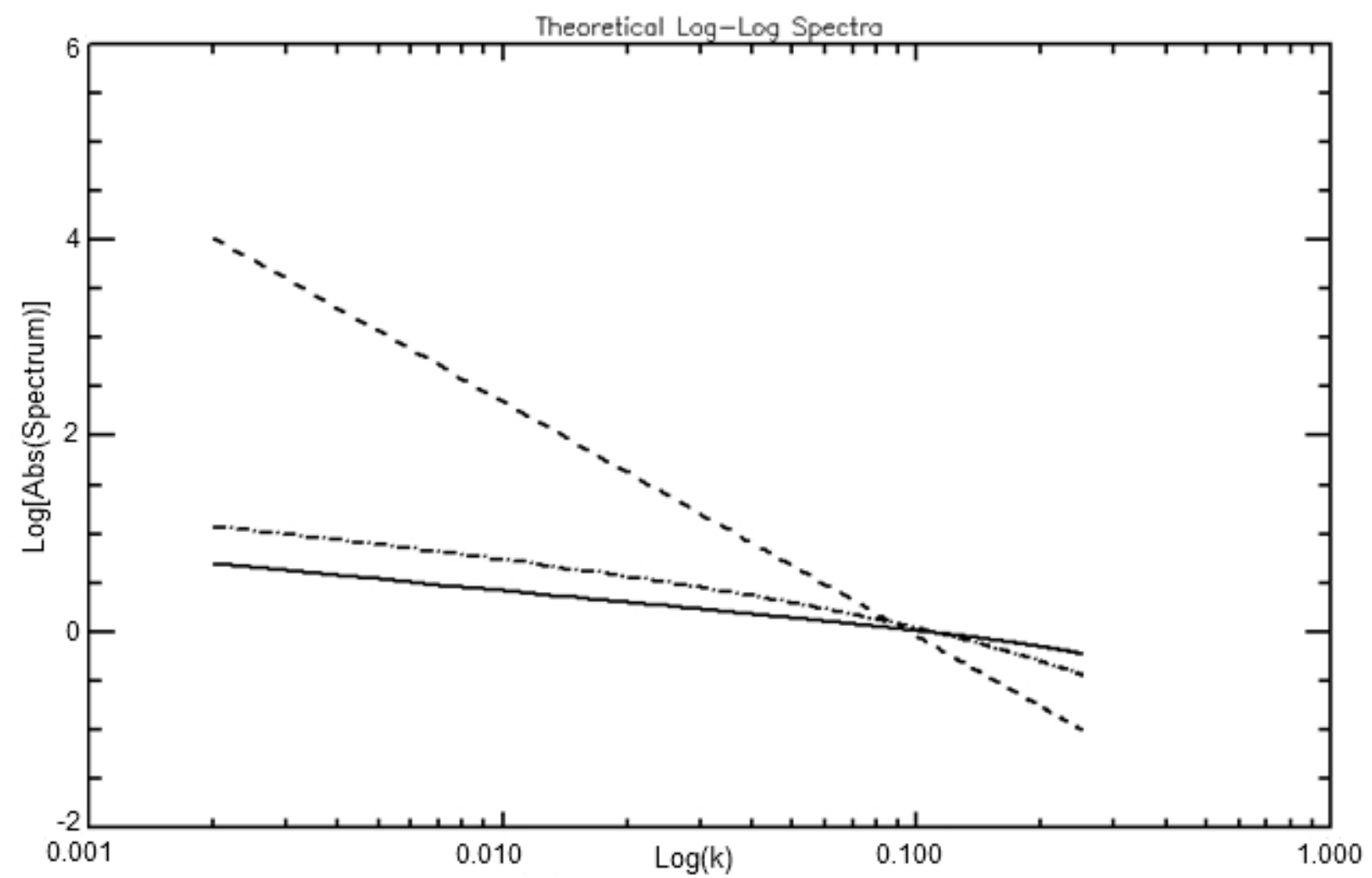

Fig. 3: Theoretical log-log plots of range (continuous line) and azimuth (dash-dot line) image cuts PSD; the dashed line represents the surface cut PSD. All the graphs are relevant to $H=0.7$. 


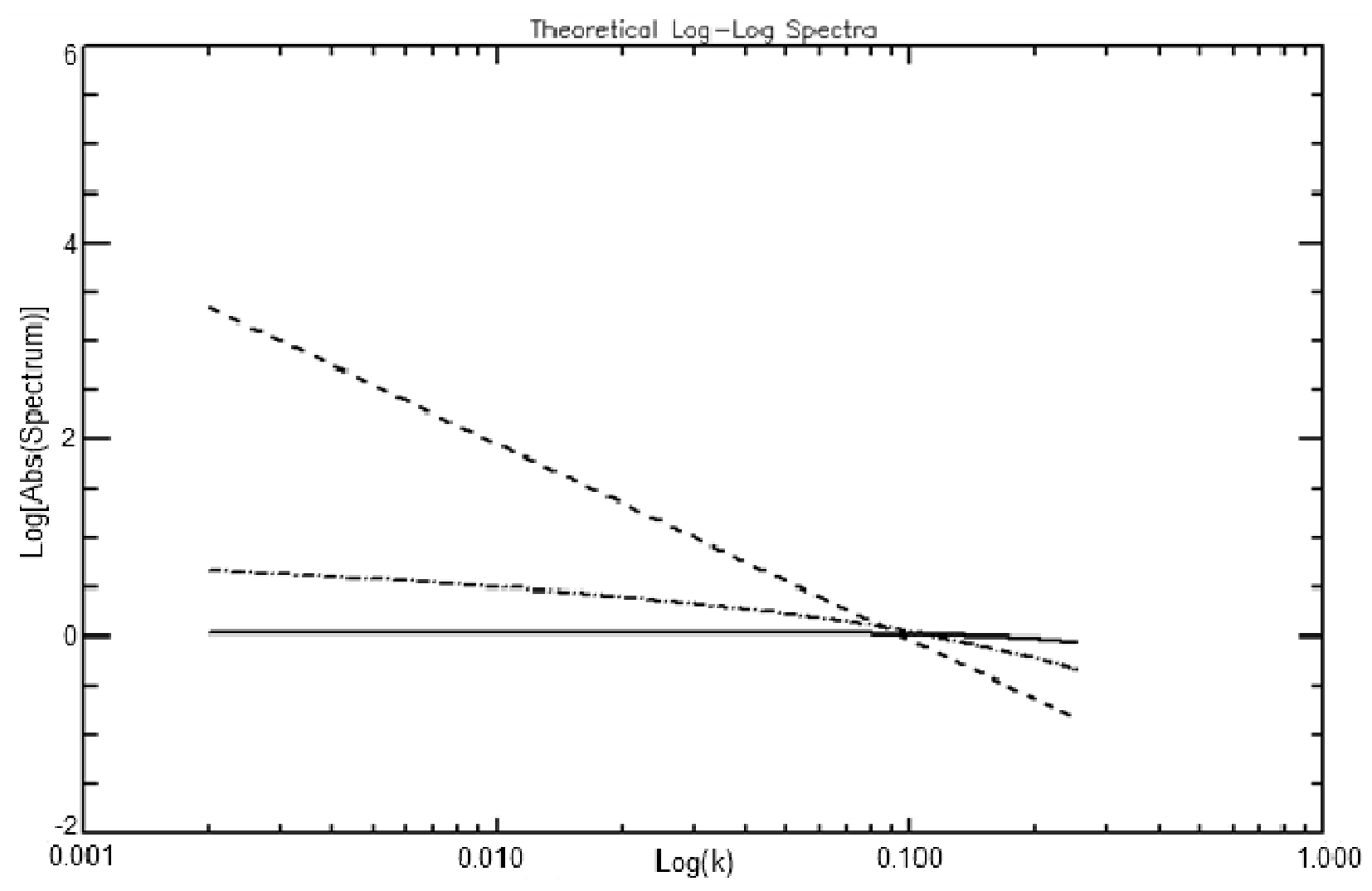

Fig.4: Theoretical log-log plots of range (continuous line) and azimuth (dash-dot line) image cuts PSD; the dashed line represents the surface cut PSD. All the graphs are relevant to $H=0.5$.

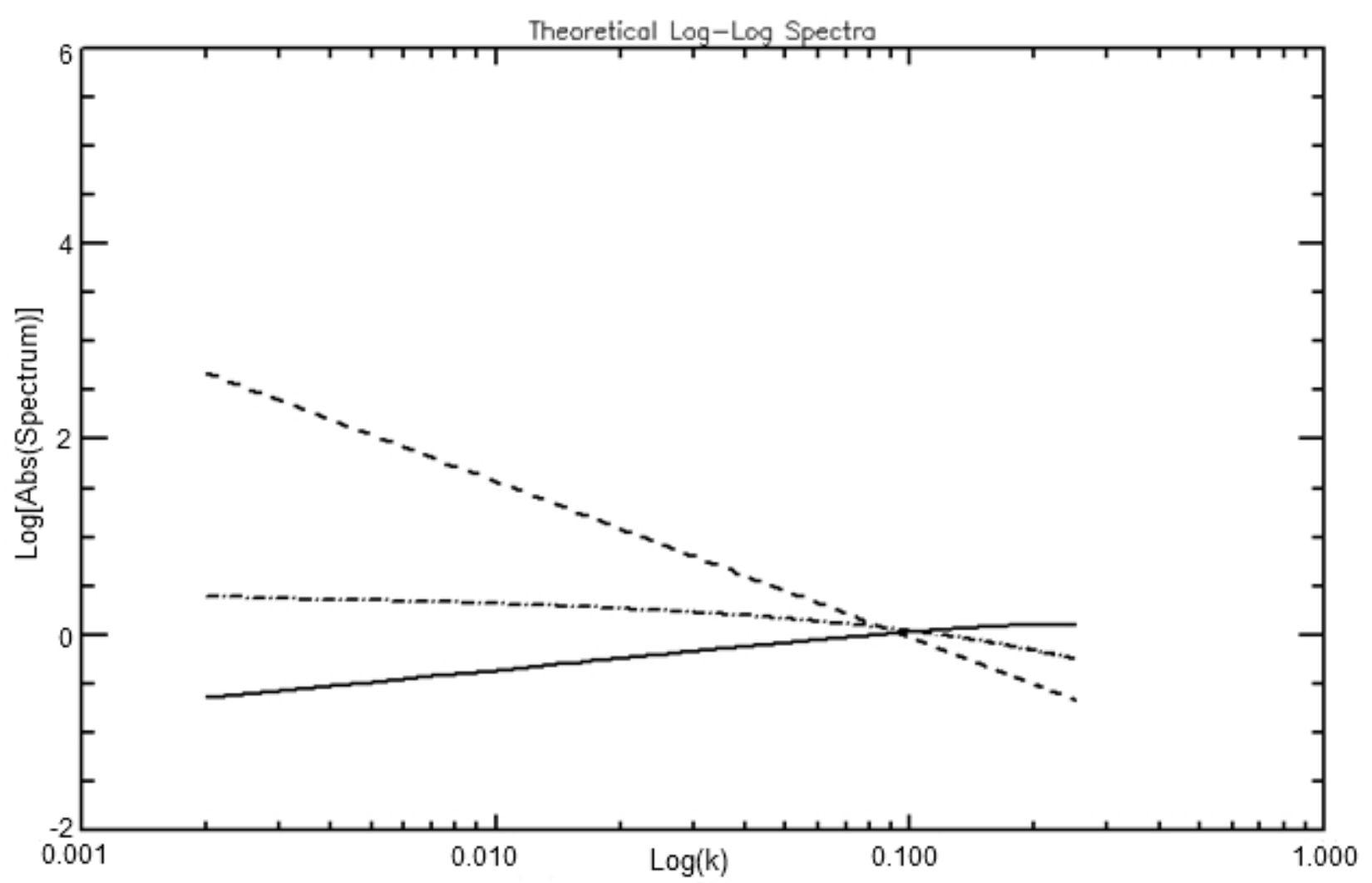

Fig.5: Theoretical log-log plots of range (continuous line) and azimuth (dash-dot line) image cuts PSD; the dashed line represents the surface cut PSD. All the graphs are relevant to $H=0.3$.

The provided figures show clearly the difference in the behaviors of range and azimuth 
image cuts PSDs. The behavior of the range cut PSD is linear in the $\log (k)-\log (|\mathrm{S}(k)|)$ plane for sufficiently low spatial frequencies, presenting a slope equal to that of the range cut PSD (dashed lines) decreased by two (compare Eq. (3.18) and (3.6)). Conversely, the plot of the azimuth cut PSD presents a more complex behavior that is, actually, not at all a power-law one.

\section{NUMERICAL SETUP}

\section{V.1 Theoretical framework validation}

In this section experimental results validating the theoretical framework developed in the previous sections are presented. In particular, in order to compare the theoretical spectra with those relevant to SAR images of fractal surfaces having controlled and known fractal parameters, we make use of the SARAS, a SAR data simulator [20].

First of all an $\mathrm{fBm}$ surface of controlled parameters is synthesized using the WeierestrassMandelbrot function [17], [30], [31]:

$$
z(x, y)=B \sum_{p=0}^{P-1} C_{p} v^{-H p} \sin \left[k_{0} v^{p}\left(x \cos \Psi_{p}+y \sin \Psi_{p}\right)+\Phi_{p}\right]
$$

wherein $B[\mathrm{~m}]$ is the overall amplitude scaling factor; $p$ is the tone index; $k_{0}\left[\mathrm{~m}^{-1}\right]$ is the wavenumber of the fundamental component (corresponding to $p=0$ ); $v>1$ is the seed of the geometric progression that accounts for spectral separation of successive tones; $0<H<1$ is the Hurst exponent; and $C_{p}, \Psi_{p}, \Phi_{p}$ are random coefficients accounting respectively for amplitude, direction, and phase of each tone. By opportunely choosing the parameters $B$ and $v$ [17], [31], the obtained Weierstrass-Mandelbrot surface is a good approximation of the required fBm surface: in Fig. 6 an $\mathrm{fBm}$ surface of parameters $H=0.8, s=0.1 \mathrm{~m}^{0.2}$ synthesized through a WeierstrassMandelbrot function is shown.

The obtained surface is used as input Digital Elevation Model (DEM) to SARAS. The type of sensor to be simulated and the scattering function must be then set. Let us note that, in this case, we work in the hypothesis that the observed surface shows the same user-defined fractal parameters at all the scales of interest (i.e., both at macroscopic and microscopic scales). We simulated an Envisat image $(\Delta x=3.986, \Delta y=19.928)$ that allows analyzing the case of extremely different resolutions in azimuth and range and, as anticipated in Section II, we choose an SPM fractal scattering model consistent with the fractal model of the surface with a VV polarization. Note that use of an $\mathrm{HH}$ polarization does not significantly change the obtained results, as previously stated in 


\section{Section III.2.}

The PSD estimation from the obtained SAR image is not a trivial issue. As a matter of fact, we deal with power-law (or power-law like) spectra that introduce unique difficulties in the spectral estimation as they suffer from both the leakage effect, that yields a spectral estimate that is insensitive to the spectral slope, and the high variance problem [32]. Among the spectral estimators available in literature, we use the Capon estimator [24], [32], [33] that is both suitable to avoid the leakage, measuring the surface spectrum accurately, and has a reduced variance, thus being very appropriate for short data records, which is the case of some of our applications. In particular, the Capon estimator performs a filtering of the spectrum consisting in discarding the low spatial frequencies (those that mainly contribute to the leakage phenomenon) and smoothing the spectrum shape in order to minimize the variance [24]. These two filtering and smoothing operations can be both controlled acting on the filter length. Moreover, since a spectral estimate derived from sampled data suffers from aliasing, if the sampled process has spectral components at frequencies greater than the Nyquist frequency, we discard all the spatial frequencies greater than $1 / 2 \Delta x$ (or $1 / 2 \Delta y$ depending on the considered cut). As an example, in Fig. 7, a non-filtered PSD is shown where the vertical axes enclose the range of wavenumbers used for estimation. In this figure the image estimated spectrum (continuous line) is compared with the theoretical one (dash-dot-dot line) computed substituting $H=0.8$ in Eq. (3.17) and the dashed and dash-dot lines mark the limit theoretical spectra, i.e. those presenting $H=0.999$ and $H=0.001$, respectively. Note, that beyond the range of values of $H$ equal to ]0,1[ the surface is not a fractal surface, as stated in Section III when the $\mathrm{fBm}$ process was introduced.

In order to compare the theoretical PSDs of a range and an azimuth cut of the image evaluated in Section II (Eq. (3.17) and (3.22), respectively) with those estimated from the SAR image, it must be taken into account that the theoretical spectra are averaged spectra. Hence, for each direction, we perform on the image several cuts sufficiently spaced one from each other to be considered uncorrelated, we estimate the spectra of these profiles using the Capon estimator and, finally, we average these spectra in order to obtain the estimated PSD. In particular, we considered 1000 sample profiles and the length of the Capon filter was set equal to 250 (a quarter of the total number of samples, as suggested in the literature on the subject [24]). Some significant results for the values of $s$ and $H$ in Table I are presented hereafter.

Moreover, by exploiting the considerations presented in the previous section concerning the PSD of a range cut of the image, a linear regression is implemented on the estimated spectra in order to retrieve the Hurst coefficient of the observed surface. In Table I such estimated values are presented together with the actual values of $H$ of the observed surface. The analysis of these results 
shows that, as far as the hypothesis of small slopes of the surface is valid, the performance of the retrieving technique is definitely good, while it starts to get worse when the aforementioned hypothesis begins to fail. More precisely, the retrieving techniques are efficient because the estimated $H$ values are so close to the actual ones to allow the discrimination of slightly different (in terms of $H$ ) surfaces from their radar images. The results presented in Table I refer to the spectra shown in Figs. 8-12, which have been evaluated using the Capon filter; in these figures the image estimated spectra are compared with the theoretical ones, as was done in Fig. 7 for a non-filtered spectrum. Moreover, in Figs. 13 and 14 two examples of the behavior of estimated azimuth spectra for two different values of $H$ are provided and compared with the theoretical one.

It is worth stressing that in this case the estimation and the regression operations are performed on profiles of 1000 samples, so that low frequency components can be easily estimated. This is not always the case when the analysis of actual SAR images is in order: in fact, in actual images, we may not have so many samples over an area presenting the same fractal parameters. Some observations about this issue are provided in the next subsection.

Table I

List OF THE PARAMETERS USED IN THE SimUlations AND SUMMARy OF RESUltS

\begin{tabular}{|c|c|c|c|}
\hline Figure number & $s$ & $H$ & Estimated value of $H$ \\
\hline Fig. 8 & 0.1 & 0.9 & 0.89 \\
\hline Fig. 9 & 0.1 & 0.8 & 0.82 \\
\hline Fig. 10 & 0.1 & 0.7 & 0.75 \\
\hline Fig. 11 & 0.1 & 0.6 & 0.64 \\
\hline Fig. 12 & 0.1 & 0.5 & 0.58 \\
\hline
\end{tabular}




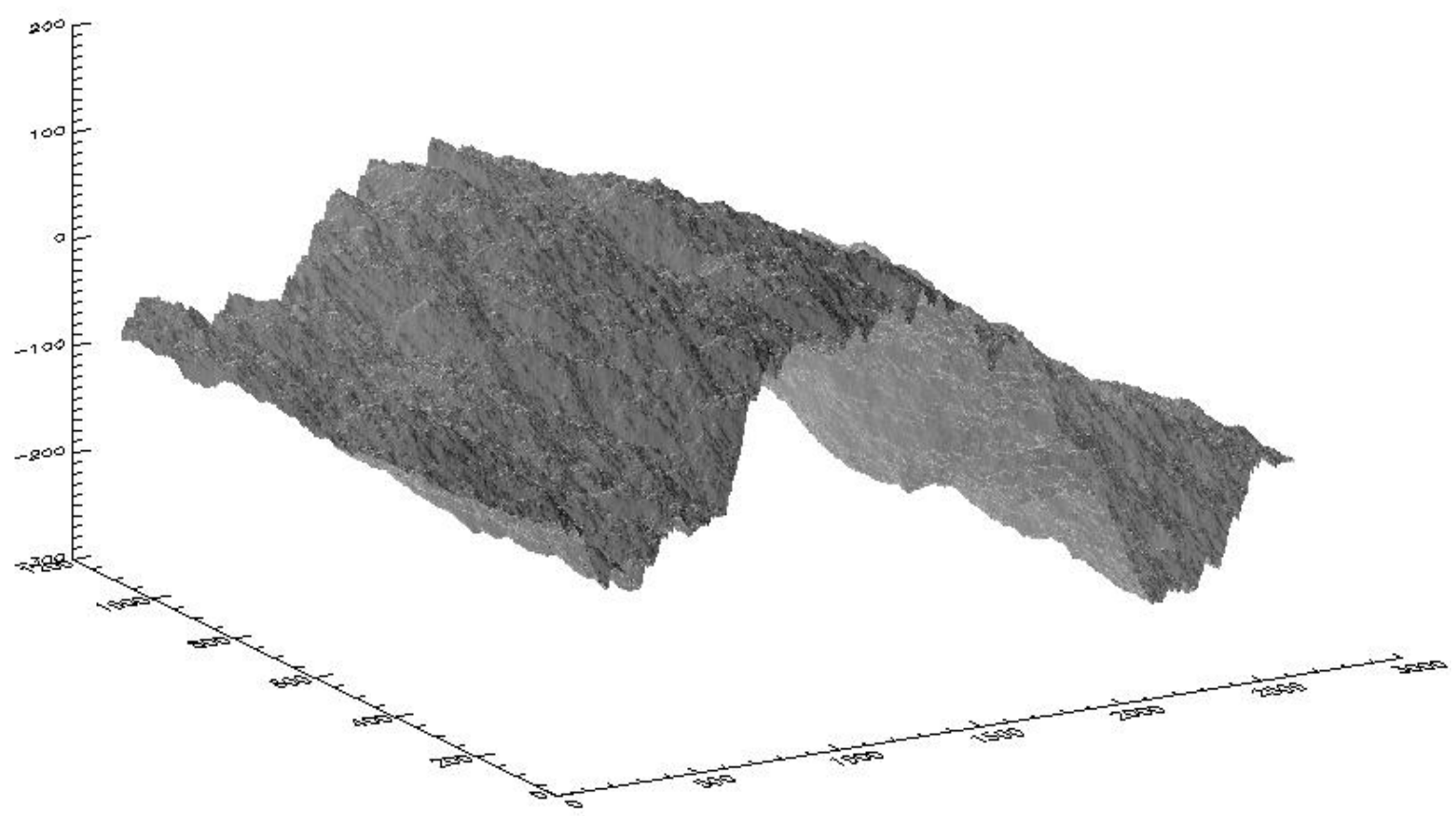

Fig. 6: Fractal surface of parameter $s=0.1 \mathrm{~m}^{0.2}, H=0.8$ synthesized through a Weierstrass-Mandelbrot function.

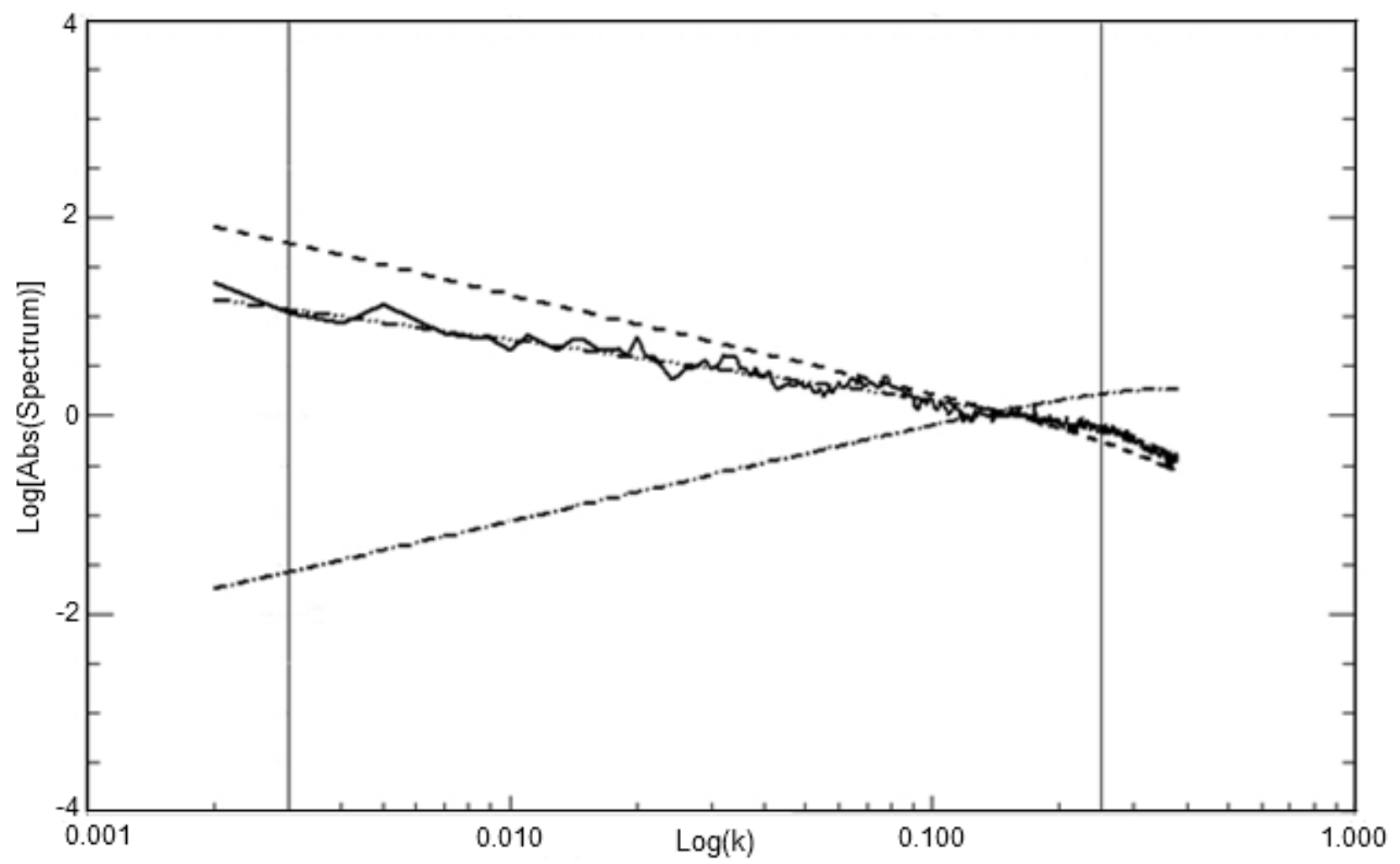

Fig. 7: Mean PSD of range cuts of the image before applying the Capon filtering (continuous line) compared with the theoretical one (dash-dot-dot line) for a fractal surface with $H=0.8$; the estimated value of $H$ is 0.86 . The theoretical spectra for $H=0.999$ (dashed line) and $H=0.001$ (dash-dot line), which represent the limit of $H$ for which a surface holds a fractal behavior, are reported. The two vertical axes mark the wavenumbers beyond which the spectrum is cut. 


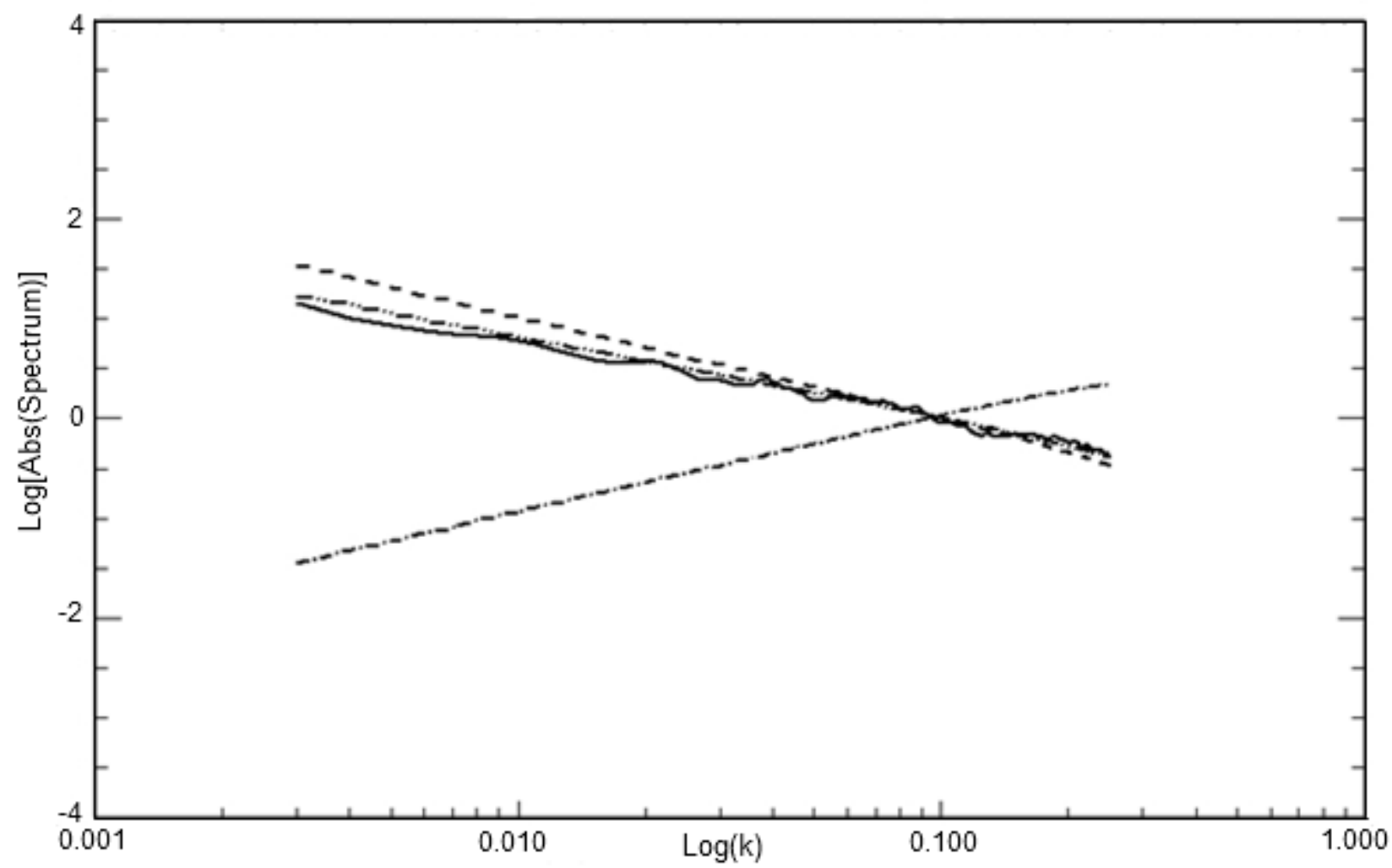

Fig. 8: Mean PSD of range cuts of the image after the application of the Capon filter (continuous line) compared with the theoretical one (dash-dot-dot line) for a fractal surface with $H=0.9$; the estimated value of $H$ is 0.89 . The theoretical spectra for $H=0.999$ (dashed line) and $H=0.001$ (dash-dot line), which represent the limit of $H$ for which a surface holds a fractal behavior, are reported. See Table I for the employed parameters.

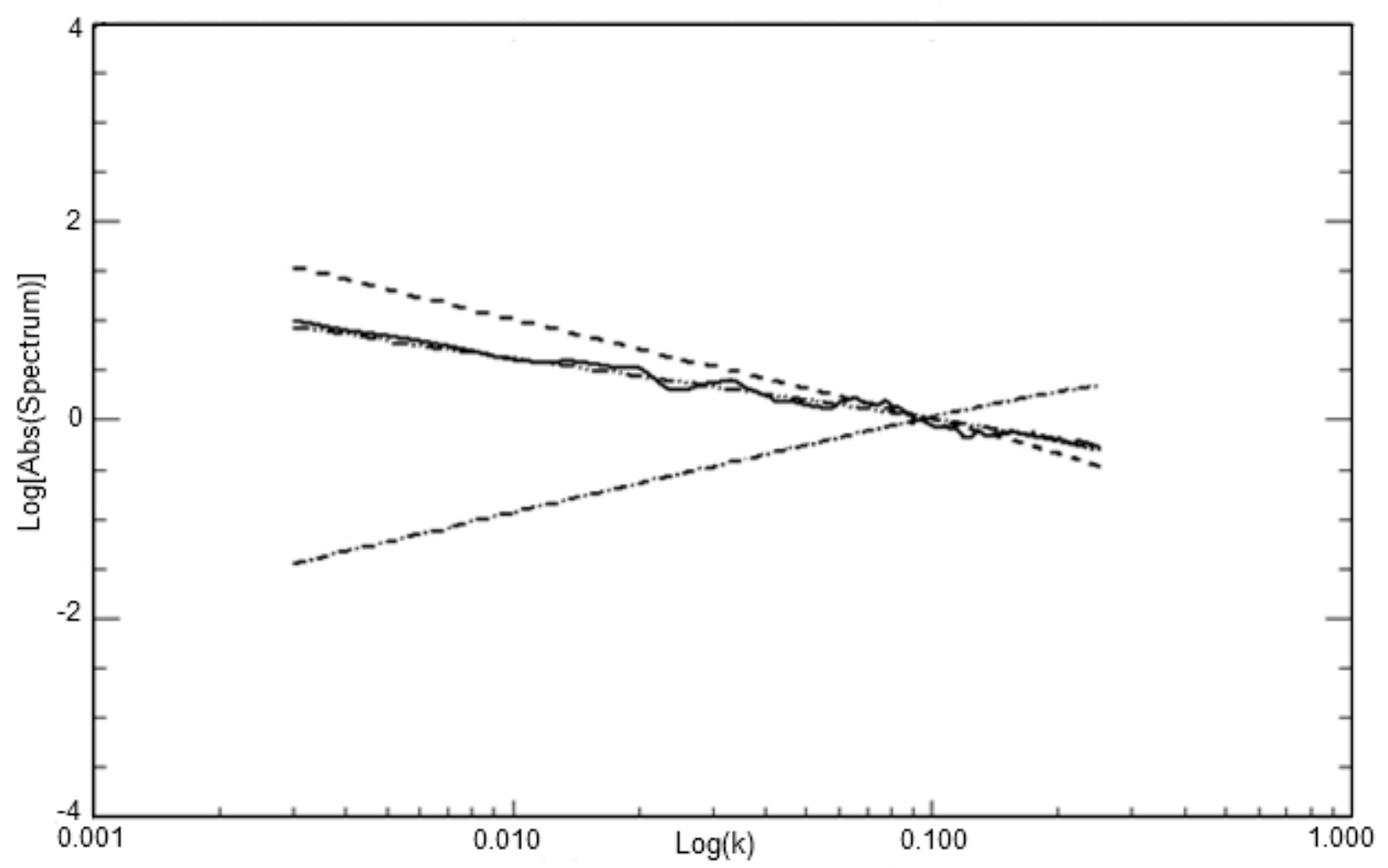

Fig. 9: Mean PSD of range cuts of the image after the application of the Capon filter (continuous line) compared with the theoretical one (dash-dot-dot line) for a fractal surface with $H=0.8$; the estimated value of $H$ is 0.82 . The theoretical spectra for $H=0.999$ (dashed line) and $H=0.001$ (dash-dot line), which represent the limit of $H$ for which a surface holds a fractal behavior, are reported. See Table I for the employed parameters. 


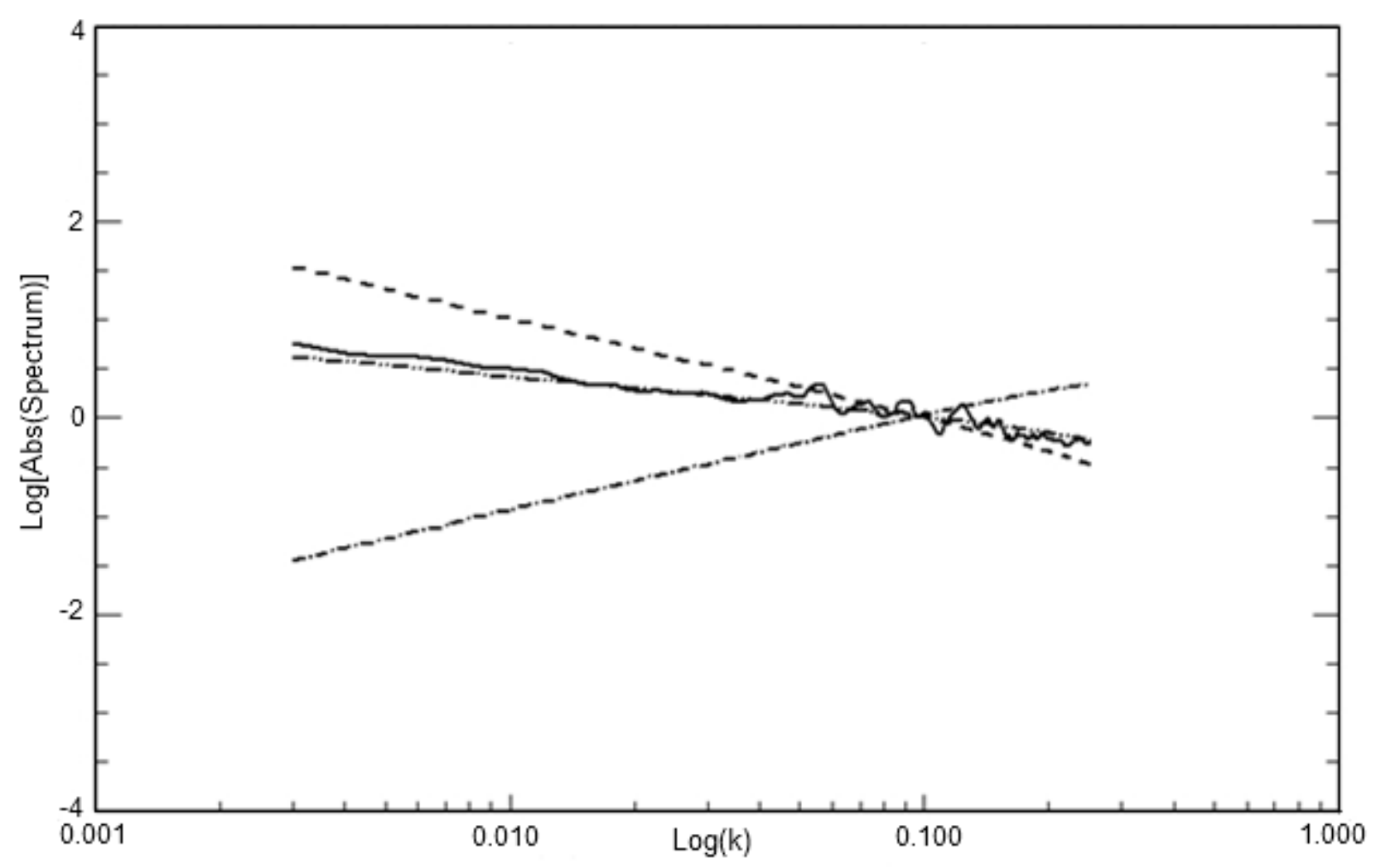

Fig. 10: Mean PSD of range cuts of the image after the application of the Capon filter (continuous line) compared with the theoretical one (dash-dot-dot line) for a fractal surface with $H=0.7$; the estimated value of $H$ is 0.75 . The theoretical spectra for $H=0.999$ (dashed line) and $H=0.001$ (dash-dot line), which represent the limit of $H$ for which a surface holds a fractal behavior, are reported. See Table I for the employed parameters.

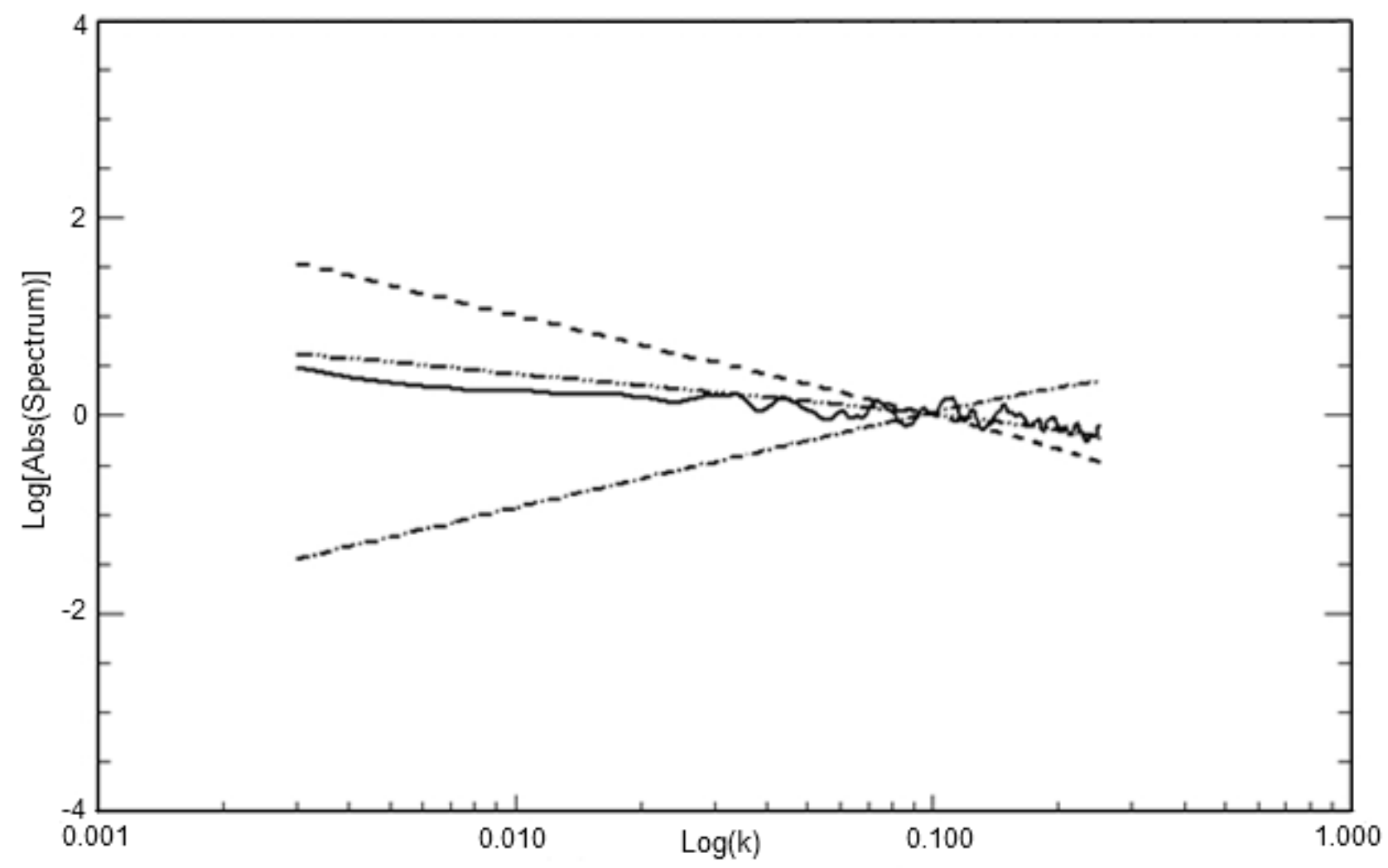

Fig. 11: Mean PSD of range cuts of the image after the application of the Capon filter (continuous line) compared with the theoretical one (dash-dot-dot line) for a fractal surface with $H=0.6$; the estimated value of $H$ is 0.64 . The theoretical spectra for $H=0.999$ (dashed line) and $H=0.001$ (dash-dot line), which represent the limit of $H$ for which a surface holds a fractal behavior, are reported. See Table I for the employed parameters. 


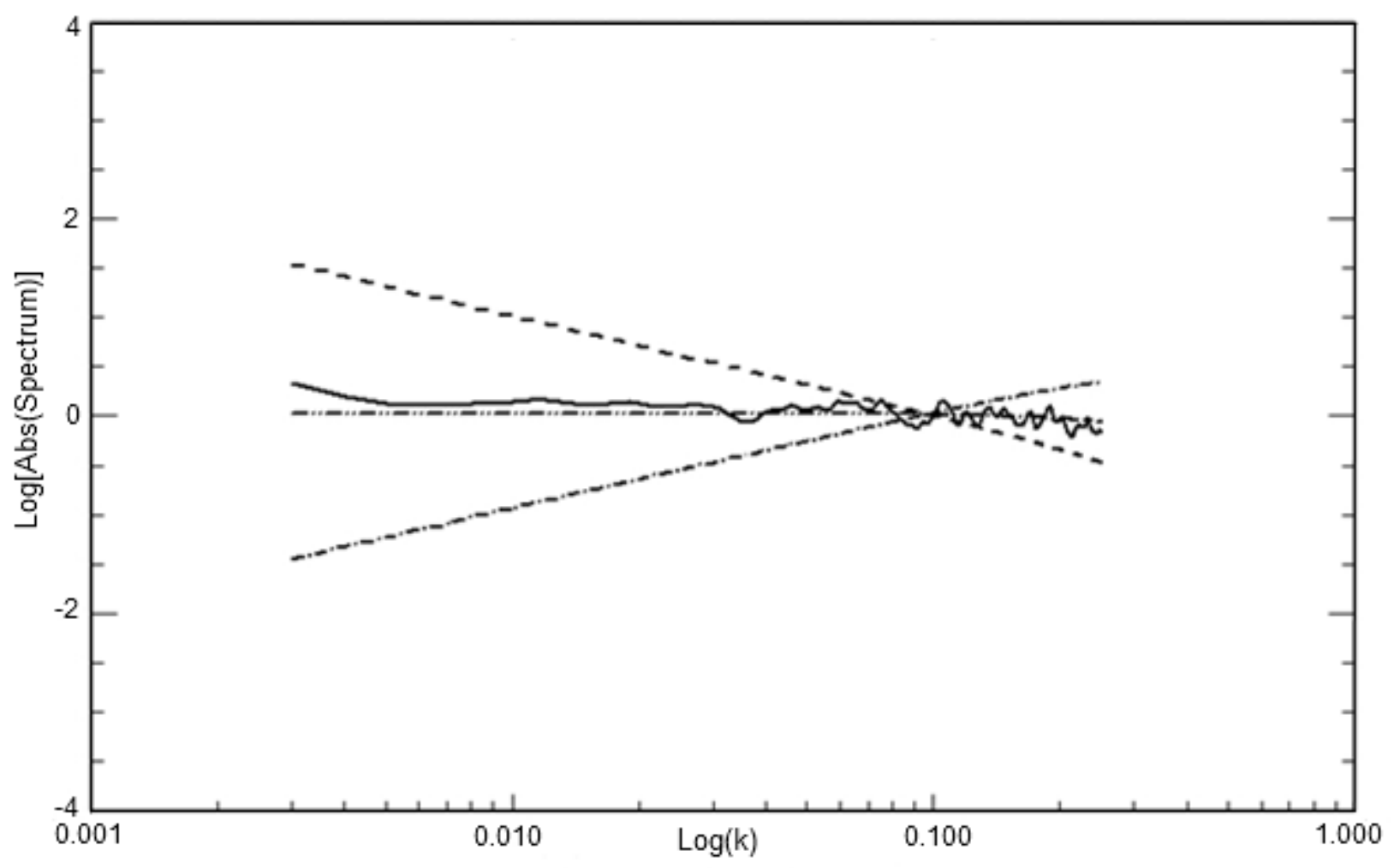

Fig. 12: Mean PSD of range cuts of the image after the application of the Capon filter (continuous line) compared with the theoretical one (dash-dot-dot line) for a fractal surface with $H=0.5$; the estimated value of $H$ is 0.58 . The theoretical spectra for $H=0.999$ (dashed line) and $H=0.001$ (dash-dot line), which represent the limit of $H$ for which a surface holds a fractal behavior, are reported. See Table I for the employed parameters.

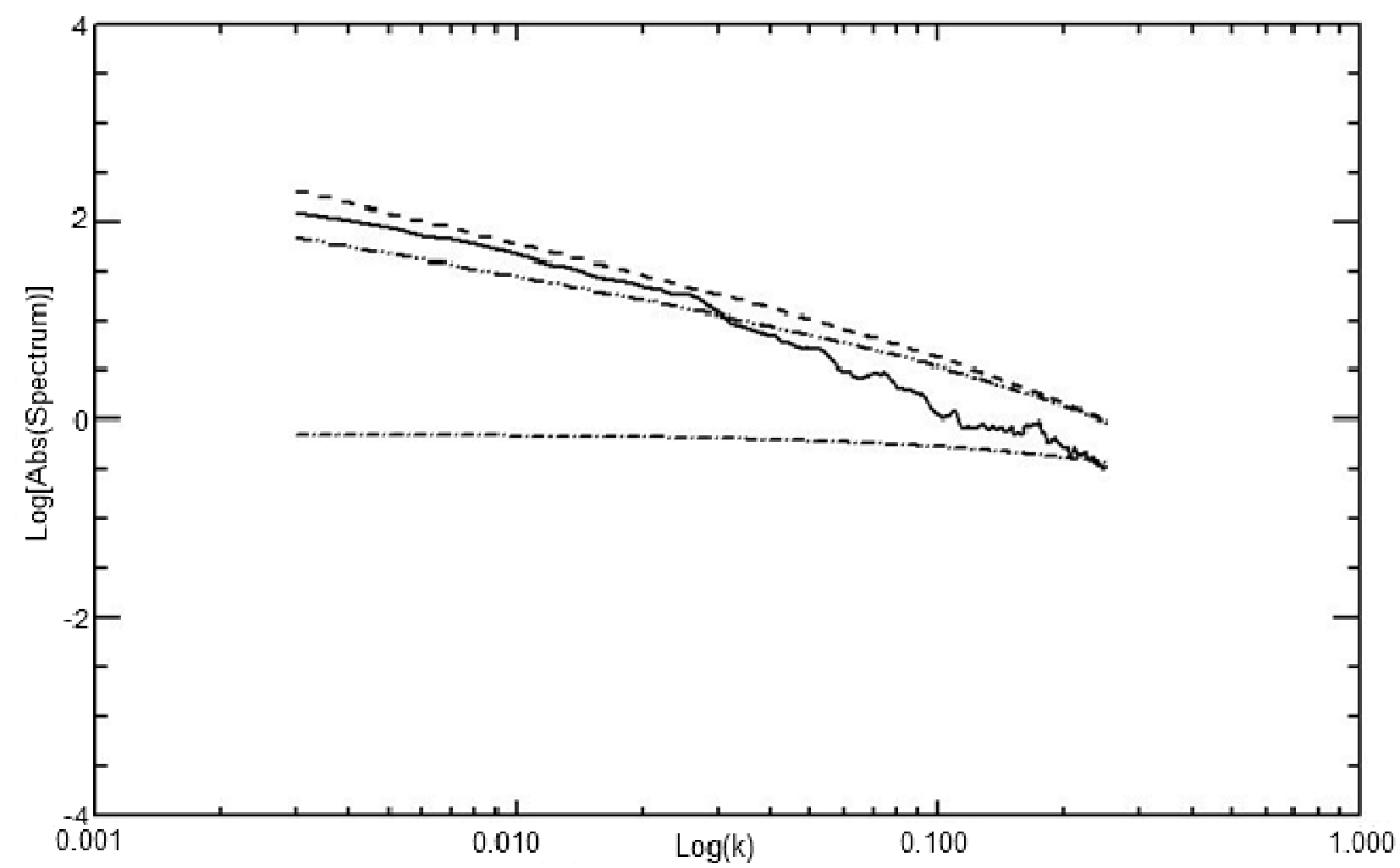

Fig. 13: Mean PSD of azimuth cuts of the image after the application of the Capon filter (continuous line) compared with the theoretical one (dash-dot-dot line) for a fractal surface with $H=0.8$. The theoretical spectra for $H=0.999$ (dashed line) and $\boldsymbol{H}=\mathbf{0 . 0 0 1}$ (dash-dot line), which represent the limit of $\boldsymbol{H}$ for which a surface holds a fractal behavior, are reported. 


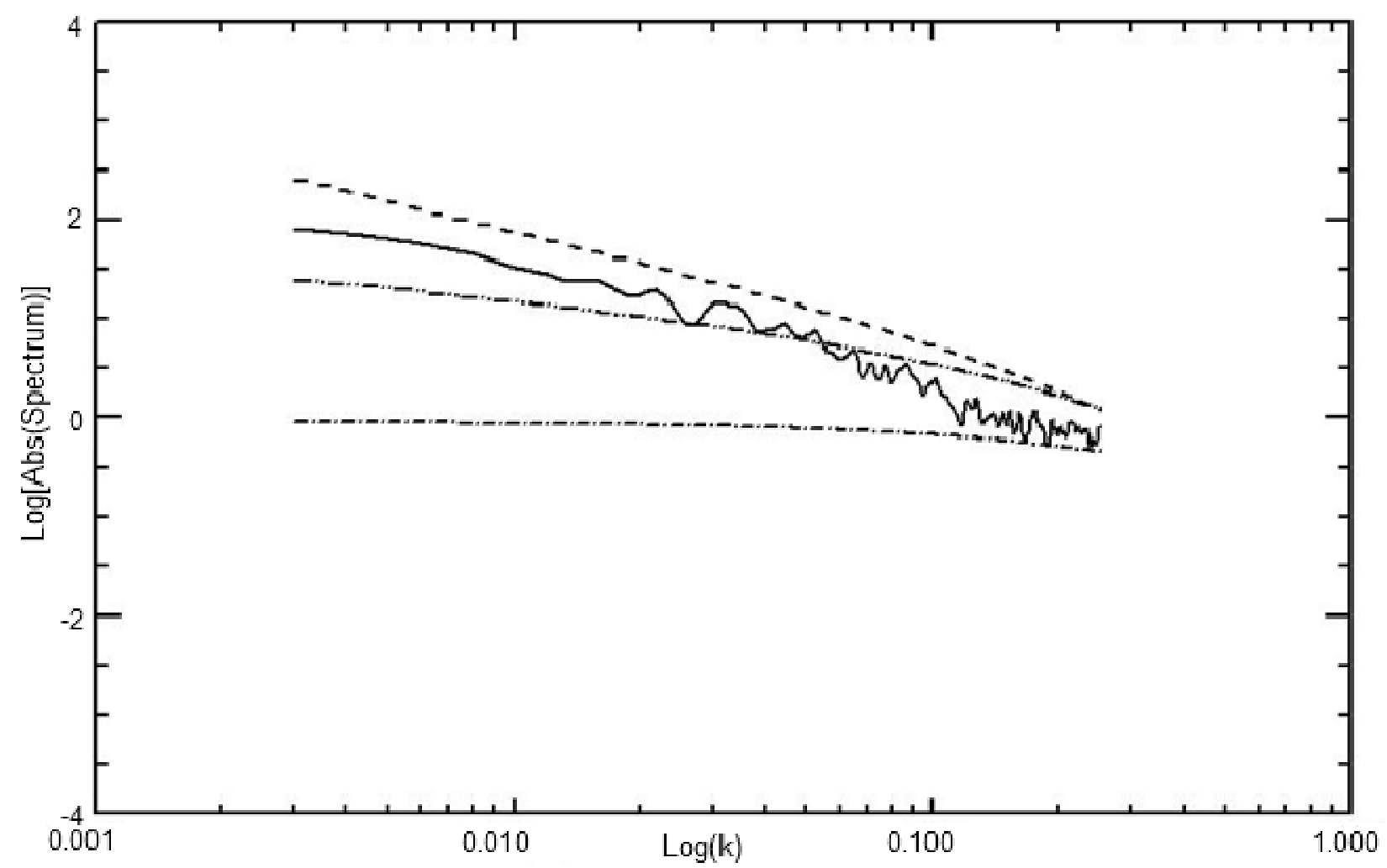

Fig. 14: Mean PSD of azimuth cuts of the image after the application of the Capon filter (continuous line) compared with the theoretical one (dash-dot-dot line) for a fractal surface with $H=0.6$. The theoretical spectra for $H=0.999$ (dashed line) and $\boldsymbol{H}=\mathbf{0 . 0 0 1}$ (dash-dot line), which represent the limit of $\boldsymbol{H}$ for which a surface holds a fractal behavior, are reported.

\section{V.2 An algorithm for the estimation of the fractal dimension from a SAR image}

In order to apply on actual SAR images the fractal retrieving technique described in the previous subsection, some considerations about the extent of the imaged area and so on the number of samples which can be used for the spectrum estimation are required. Note that, only for the sake of an easier comprehension of the physical role of the fractal parameter at stake, in the present subsection, instead of retrieving the Hurst coefficient, $H$, we extract, from the SAR image, the fractal dimension $D$ of the observed scene. The retrieving of $D$ and that of $H$ are completely equivalent, being the two parameters related through the Eq. (3.2). The values of $D$, nevertheless, make intuitively more immediate the recognition of the surface roughness, as it express the topographic fractal dimension of the surface of interest.

In Section V.1, in order to validate the theoretical results, simulated profiles of 1000 samples, holding the same fractal dimension at all scales, have been considered. Obviously, an actual SAR image of a natural area can present appreciable variations of the fractal dimension over the observed scene. Indeed, the fractal dimension is a local characteristic of the surface and an effective technique for the retrieving of the fractal dimension should work on small homogeneous patches of the image. Therefore, in order to obtain a map of the fractal dimension starting from a SAR image, i.e. a matrix of the point by point estimated fractal dimension of the observed scene, a 
specific algorithm, based on the inversion and estimation logic described in the previous section, has been implemented. The proposed algorithm makes use of a sliding window which, spanning the entire image, performs in each iteration the retrieving of $D$ as described in the previous sub-section. The choice both of the sliding window dimensions and of the number of range cut spectra averaged in each window in order to obtain the mean PSD, depends on the specific needs of the user and results from a trade-off between estimation accuracy, computational time and resolution of the output fractal dimension map. As a matter of fact the choice of a larger window allows obtaining a more accurate estimate of the fractal dimension (depending on the number of samples drawn in the range direction) but makes the resolution of the final fractal map worse and increases the computational time. Concerning the number of range cut spectra averaged in each window, the larger this number the better the PSD estimation, even if the computational time increases: in particular, this is true if we assume that all the considered range cuts in the window pertain to the same type of terrain.

In order to evaluate the performance of this algorithm a test on a canonical fractal case is presented: the algorithm has been applied to a simulated (canonical) speckle-free (corresponding to an infinite number of looks) SAR image obtained as described in the previous section (i.e. providing as input to the simulator a DEM of a surface holding the same fractal parameters at all scales) with several dimensions of the elaboration window. In Fig.15 the simulated Envisat image of 1000x 1000 pixels of a natural surface with fractal parameters $D=2.2, s=0.1 \mathrm{~m}^{0.2}$ is shown. In Fig. 16, 17 and 18 the corresponding fractal maps obtained using windows of $51 \times 51$ pixels, $35 \times 35$ pixels, $21 \times 21$ pixels, respectively, are presented. In Table II the statistics of these fractal maps are summarized: in particular, the mean and the standard deviation of the estimated fractal dimension are provided. 


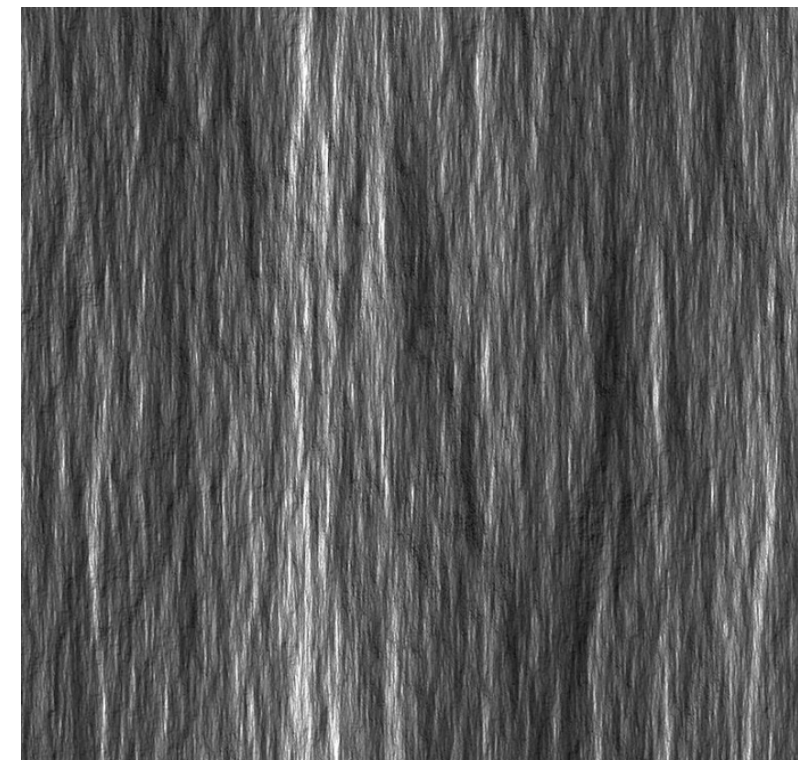

Fig. 15: Simulated SAR Image of a canonical surface of fractal parameters $s=0.1, D=2.2$

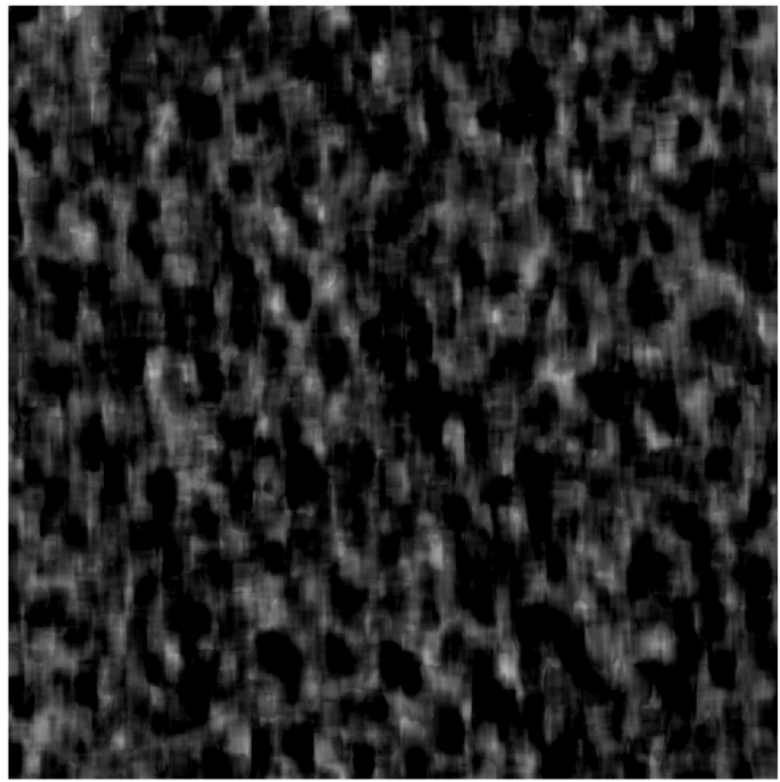

Fig. 17: Fractal map relevant to the SAR image in Fig.14 using a sliding window of dimension $35 \times 35$ pixels

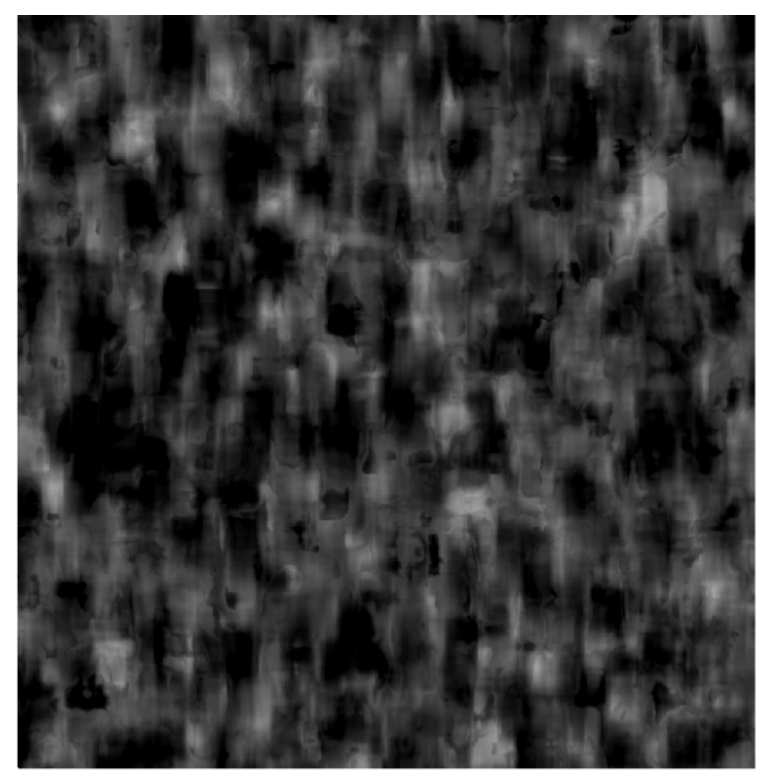

Fig. 16: Fractal map relevant to the SAR image in Fig.14 using a sliding window of dimension $51 \times 51$ pixels

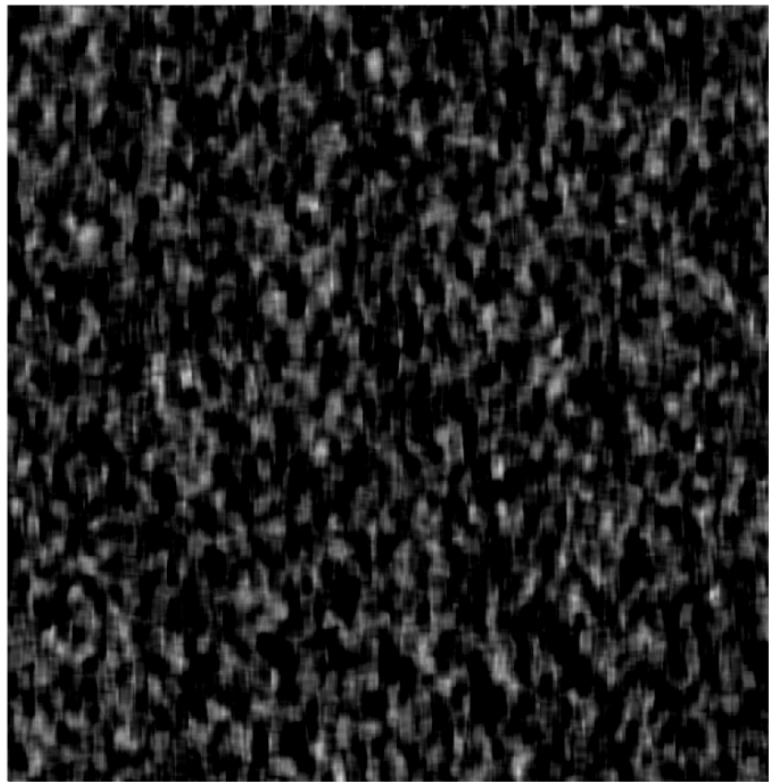

Fig. 18: Fractal map relevant to the SAR image in Fig.14 using a sliding window of dimension $21 \times 21$ pixels

Table II

Statistics of the Fractal Maps (SPEckle Free CaSe)

\begin{tabular}{|c|c|c|c|}
\hline Figure number & $\begin{array}{c}\text { Sliding window } \\
\text { dimensions }\end{array}$ & $D$ mean & $D$ standard deviation \\
\hline Fig. 16 & $51 \times 51$ pixels & 2.19 & 0.13 \\
\hline Fig. 17 & $35 \times 35$ pixels & 2.11 & 0.16 \\
\hline Fig. 18 & $21 \times 21$ pixels & 2.06 & 0.19 \\
\hline
\end{tabular}


Finally, in order to evaluate the performance of this type of post-processing on SAR images affected by the speckle phenomenon, the algorithm has been applied to simulated SAR images generated by the SARAS and taking into account the speckle effect. In this case, a sliding window of 51x51 pixels has been used. As shown in Figs. 19-22 and summarized in Table III, the presence of speckle does not significantly invalidate the effectiveness of the estimation for the considered cases: in particular, four simulated SAR images have been considered, for which the observed surface presents different values of $D$ and $s$, as summarized in Table III. As a matter of fact, the presented spectrum estimation technique performs a sort of intrinsic speckle filtering. First of all, averaging several spectra relevant to range cuts sufficiently spaced in the azimuth direction to be considered uncorrelated, implies a significant mitigation of the speckle effect. Besides, as a result of the anti-aliasing filtering, which consists in discarding the high frequencies components of the spectrum (see Section V.1), the range of frequencies mostly affected by the speckle is discarded. Anyway, note that the case of speckle is subject of current investigation and the study proposed here is only a preliminary one: hence, an exhaustive study of this phenomenon, where a more significant range of window dimensions and fractal parameters should be considered, is beyond the scope of this paper.

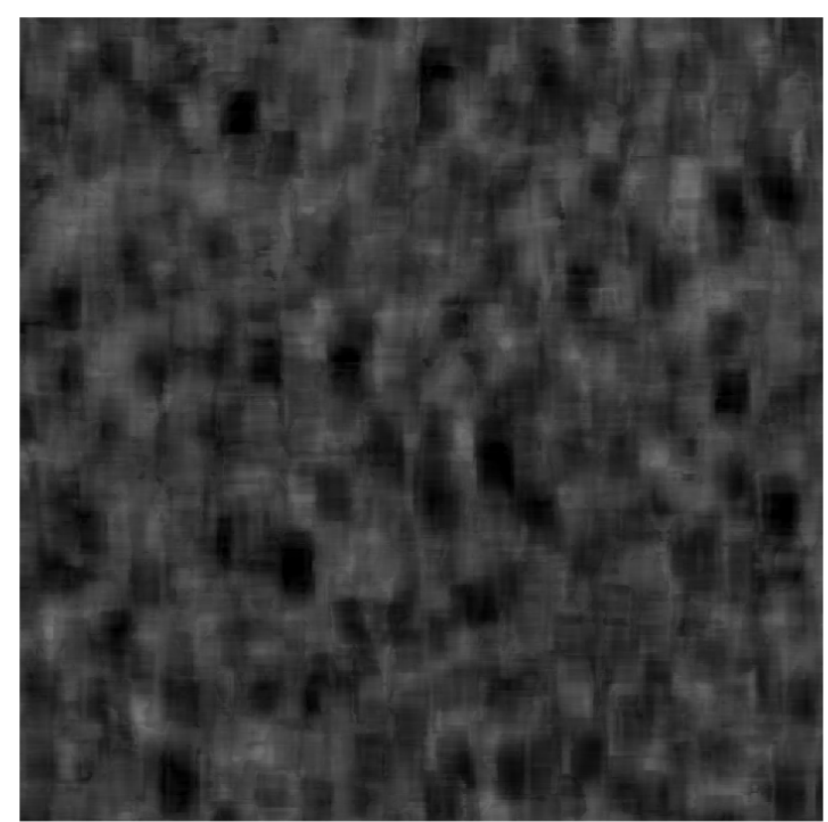

Fig. 19 Fractal maps relevant to SAR Images of fractal parameters in Table III in presence of speckle.

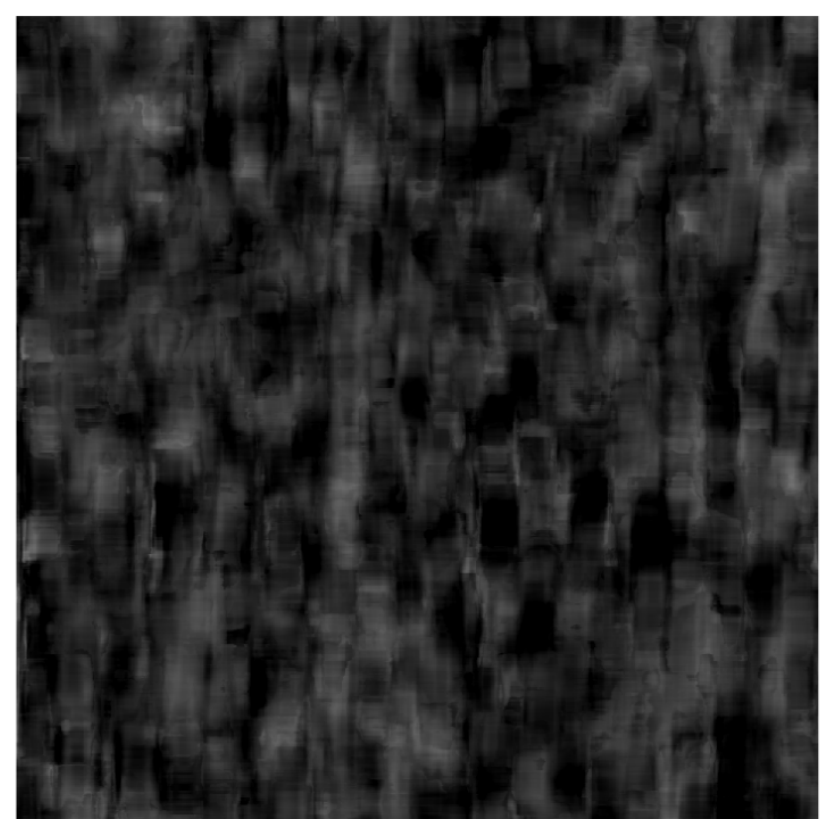

Fig. 20 Fractal maps relevant to SAR Images of fractal parameters in Table III in presence of speckle. 


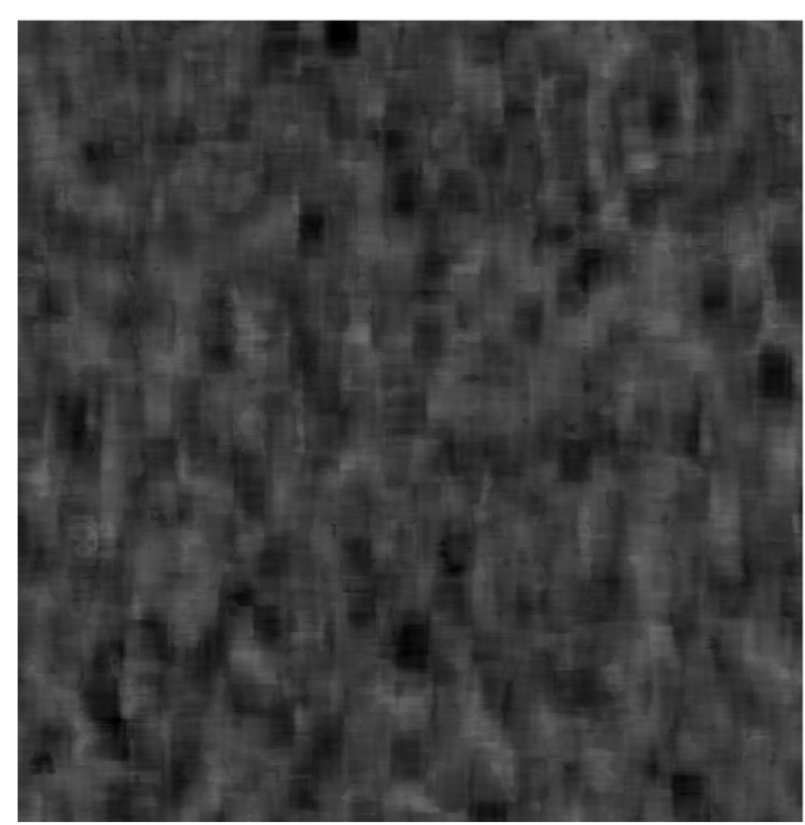

Fig. 21 Fractal maps relevant to SAR Images of fractal parameters in Table III in presence of speckle.

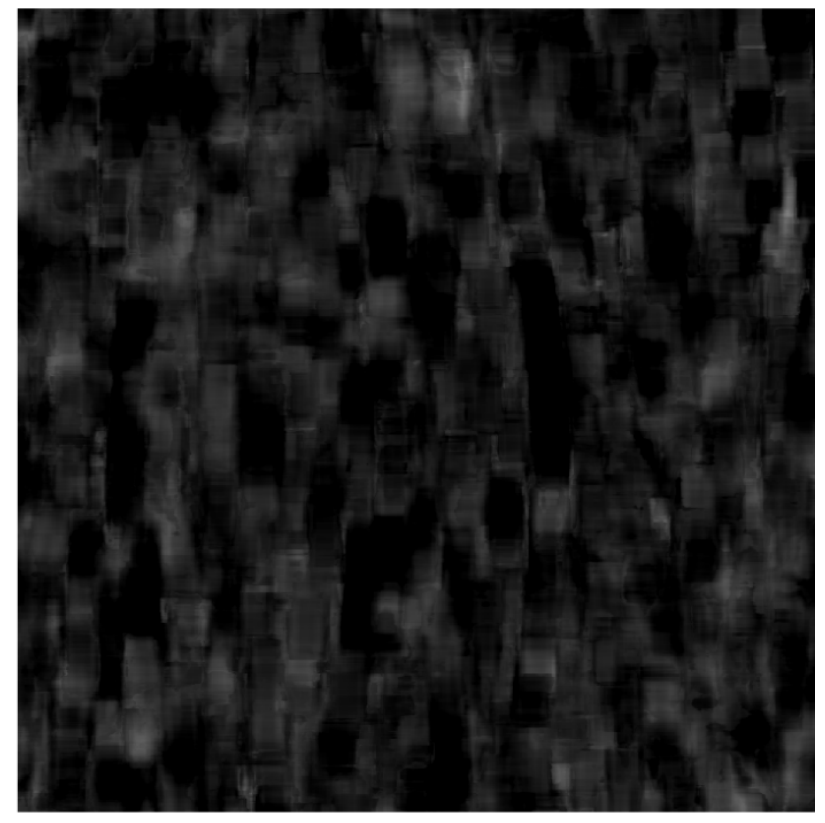

Fig. 22 Fractal maps relevant to SAR Images of fractal parameters in Table III in presence of speckle.

Table III

Statistics of the Fractal Maps (SPeckle CaSe)

\begin{tabular}{|c|c|c|c|c|}
\hline $\begin{array}{c}\text { Figure } \\
\text { number }\end{array}$ & $\begin{array}{c}s\left[\mathrm{~m}^{1-H}\right] \text { of the imaged } \\
\text { surface }\end{array}$ & $\begin{array}{c}D \text { of the imaged } \\
\text { surface }\end{array}$ & $D$ mean & $\begin{array}{c}D \text { standard } \\
\text { deviation }\end{array}$ \\
\hline Fig. 19 & 0.3 & 2.3 & 2.22 & 0.07 \\
\hline Fig. 20 & 0.3 & 2.1 & 2.14 & 0.08 \\
\hline Fig. 21 & 0.5 & 2.3 & 2.23 & 0.06 \\
\hline Fig. 22 & 0.5 & 2.1 & 2.08 & 0.08 \\
\hline
\end{tabular}

\section{CONCLUSIONS}

In this paper a complete direct model of the SAR imaging process for fractal surfaces has been presented for the first time. Furthermore, a post-processing technique based on this model has been developed and tested on simulated SAR images.

The proposed model, which is in turn based on sound radar, electromagnetic and geometrical models, links, in an analytical way, the SAR image of a natural surface to the parameters that quantitatively describe the surface of interest. In particular, due to the fact that a natural surface presents an inherent stochastic behavior, we computed in closed form, under the hypothesis of small slopes of the surface, the statistical characterization of the acquired image, that depends on the parameters used for the surface characterization. For the description of the natural 
scenes fractal models have been used, as they are widely recognized in literature as the best ones to represent the roughness of this type of areas. Moreover, in order to deal with the scattering problem, fractal models that take into account the interaction of the electromagnetic field with a fractal surface, have been used too. Therefore the framework of the presented SAR imaging model is, for the first time, completely fractal based.

The main theoretical results presented in this paper can be summarized as follows:

- it has been demonstrated that the reflectivity of the imaged natural scene linearly depends, in the hypothesis of small slopes, only on the partial derivative along the range direction of the two-dimensional surface;

- after adequately evaluating the range derivative process of the surface, the two-dimensional autocorrelation function of the reflectivity and of the SAR image have been computed in closed form;

- starting from the two-dimensional autocorrelation function, the Power Spectral Densities of a range and an azimuth cut of the image have been computed in closed form; the range and azimuth PSDs show very different behaviors, analytically highlighting the intrinsic asymmetry of a SAR image: in particular, neither of them is rigorously fractal but the range one, in an appropriate range of wavenumbers, presents a power-law behavior with a slope related to the fractal dimension of the observed surface;

- performing linear regression techniques on the PSD of range cuts of the SAR image the fractal dimension $D$ of the observed natural surface can be retrieved.

The experimental framework of this paper consists in two main aspects: on the one hand the numerical validation of the theoretical results, while on the other hand, a first practical application of the proposed technique, consisting in a post-processing of the SAR image for the estimation of the fractal dimension. First of all, a complete simulation chain allowing the generation of a SAR image of a fractal surface of known parameters has been implemented. On the simulated image an algorithm performing the inversion of the theoretical model has been applied: the PSDs of range and azimuth cuts of the image have been estimated and then compared with the theoretical ones. Performing a linear regression on the range spectrum, the fractal dimension of the imaged surface has been estimated and then compared with the actual one. All the experimental results show a very good agreement with the theoretical ones.

Finally, an innovative post-processing of the SAR image providing a map of the point by point fractal dimension of the observed scene has been presented. It has been applied to simulated SAR images of canonical fractal surfaces both without and with the speckle effect. This kind of elaboration will allow the generation of fractal maps from amplitude only SAR images and the 
development of several kinds of value added products for SAR images of natural scenes.

\section{ACKNOWLEDGEMENT}

This work has been supported by the Agenzia Spaziale Italiana (ASI) within the COSMO-SkyMed AO projects "Exploitation of fractal scattering models for COSMO-SkyMed images interpretation", project id. 1200, and "Buildings Feature Extraction from Single SAR Images: Application to COSMO-SkyMed High Resolution SAR Images", project id. 2202.

\section{REFERENCES}

[1] G. Di Martino, A. Iodice, D. Riccio, and G. Ruello, "A novel approach for disaster monitoring: fractal models and tools," IEEE Trans. Geosci. Remote Sens., vol. 45, no. 6, pp. 1559-1570, Jun. 2007.

[2] Voigt, S.; Kemper, T.; Riedlinger, T.; Kiefl, R.; Scholte, K.; Mehl, H.; , "Satellite Image Analysis for Disaster and Crisis-Management Support," Geoscience and Remote Sensing, IEEE Transactions on, vol.45, no.6, pp.1520-1528, June 2007.

[3] S. Aksoy, H. G. Akcay, and T. Wassenaar, "Automatic mapping of linear woody vegetation features in agricultural landscapes using very high resolution imagery," IEEE Trans. Geosci. Remote Sens., vol. 48, no. 1, pp. 511-522, Jan. 2010.

[4] Campbell, B.A., "Scale-dependent surface roughness behavior and its impact on empirical models for radar backscatter", IEEE Trans. Geosci. Remote Sens., 47, 3480-3488, Oct. 2009.

[5] A. Pentland, "Fractal-based description of natural scene," IEEE Trans. Pattern Anal. Mach. Intell., vol. PAMI-6, no. 6, pp. 661-674, Nov. 1984.

[6] P. Kube and A. Pentland, "On the imaging of fractal surfaces," IEEE Trans. Pattern Anal. Mach. Intell., vol. 10, no. 5, pp. 704-707, Sep. 1988.

[7] G. Korvin, "Is the optical image of a non-Lambertian fractal surface fractal?" IEEE Geosci. Remote Sens. Lett., vol. 2, no. 4, pp. 380-383, Oct. 2005.

[8] L.M. Kaplan, "Extended Fractal Analysis for Texture Classification and Segmentation," IEEE Trans. Image Processing, vol. 8, no. 11, pp. 1572-1585, Nov. 1999.

[9] P. Addesso, S. Marano, R. Restaino, and M. Tesauro, "Correlation properties of signals backscattered from fractal profiles," IEEE Trans. Geosci. Remote Sens., vol. 45, no. 9, pp. 28592868, Sep. 2007.

[10] Yen-Ching Chang; Shyang Chang; , "A fast estimation algorithm on the Hurst parameter of discrete-time fractional Brownian motion," Signal Processing, IEEE Transactions on, vol.50, no.3, pp.554-559, Mar 2002.

[11] W. Dierking, "Quantitative roughness characterization of geological surfaces and implications 
for radar signature analysis," IEEE Trans. Geosci. Remote Sens., vol. 37, no. 5, pp. 2397 - 2412, Sep. 1999.

[12] Pesquet-Popescu, B.; Vehel, J.L.; , "Stochastic fractal models for image processing," IEEE Signal Processing Magazine, vol.19, no.5, pp. 48- 62, Sep 2002.

[13] B.B. Mandelbrot, The Fractal Geometry of Nature. New York: Freeman, 1983.

[14] K. Falconer, Fractal Geometry. Chichester, U.K.: Wiley, 1989.

[15] J.S. Feder, Fractals. New York: Plenum, 1988.

[16] G. Franceschetti, A. Iodice, and D. Riccio, "Fractal models for scattering from natural surfaces," in Scattering, R. Pike and P. Sabatier, Eds. London, U.K.: Academic, Sep. 2001, pp. $467-485$.

[17] G. Franceschetti, D. Riccio, Scattering, Natural Surfaces and Fractals. Academic Press, Burlington (MA), USA, 2007.

[18] G. Franceschetti, A. Iodice, M. Migliaccio, D. Riccio, "Scattering from natural rough surfaces modeled by fractional Brownian motion two-dimensional processes", IEEE Trans. Antennas Prop., vol. 47, no. 9, pp. 1405-1415, Sep. 1999.

[19] G. Di Martino, A. Iodice, D. Riccio, G. Ruello, "Imaging of Fractal Profiles", IEEE Trans. Geosci. Remote Sens., vol. 48, no. 8, pp. 3280 - 3289, Aug. 2010.

[20] G. Franceschetti, M. Migliaccio, D. Riccio, G. Schirinzi, "SARAS: a SAR Raw Signal Simulator", IEEE Trans. Geosci. Remote Sens., vol. 30, no. 1, pp. 110-123, Jan.1992.

[21] G. Franceschetti, R. Lanari, Synthetic Aperture Radar Processing. CRC Press, New York, 1999.

[22] P. Flandrin, "On the spectrum of Fractional Brownian Motion". IEEE Trans. Inform. Theory, 35, 197-199, Jan. 1989.

[23] B. B. Mandelbrot, J. W. Van Ness, "Fractional Brownian motions, fractional noises and applications", SIAM Rev., vol. 10, no. 4, pp. 422-437, Oct. 1968.

[24] T. Austin, A. W. England, G. H. Wakefield, "Special problems in the estimation of power-law spectra as applied to topographical modeling", IEEE Trans. Geosci. Remote Sens., vol. 32, no. 4, pp. 928-939, July 1994.

[25] M. K. Shepard, B. A. Campbell, M. H. Bulmer, T. G. Farr, L. R. Gaddis, and J. J. Plaut, "The roughness of natural terrain: A planetary and remote sensing perspective", J. Geophys. Res., 106, 32,777-32,795, Dec. 2001.

[26] Gaddis, L.R., Mouginis-Mark, P.J. and Hayashi, J.N., "Lava flow surface textures: SIR-B radar image texture, field observations, and terrain measurements" Photogrammetric Eng. Remote Sens., 56, 211-224, Feb. 1990.

[27] B.B. Mandelbrot, Gaussian Self-Affinity and Fractals. Springer Verlag, New York, 2001. 
[28] I. M. Gelfand and G. E. Shilov, Generalized Functions. Burlington, MA: Academic, 1964.

[29] I. S. Gradshteyn and I.M. Ryzhik, Table of Integrals, Series and Products. New York: Academic, 1980.

[30] G. Ruello, P. Blanco, A. Iodice, J. J. Mallorqui, D. Riccio, A. Broquetas, and G. Franceschetti, "Synthesis, construction and validation of a fractal surface," IEEE Trans. Geosci. Remote Sens., vol. 44, no. 6, pp. 1403-1412, June 2006.

[31] M. V. Berry and Z. V. Lewis, "On the Weierstrass-Mandelbrot fractal function," Proc. R. Soc. Lond. A, Math. Phys. Sci., vol. 370, no. 1743, pp. 459-484, Apr. 1980.

[32] S. M. Kay, Modern Spectral Estimation. Englewood Cliffs, NJ: Prentice- Hall, 1988.[32] J. Capon, "High-resolution frequency-wavenumber spectrum analysis," Proceedings of the IEEE , vol.57, no.8, pp. 1408- 1418, Aug. 1969.

[33] J. Capon, "High-resolution frequency-wavenumber spectrum analysis", Proc. IEEE, vol. 57, no. 8, pp. $1408-1418,1969$. 